\title{
On the Two-Level Uncapacitated Facility Location Problem
}

\author{
Karen Aardal \\ Department of Computer Science, Utrecht University \\ Utrecht, The Netherlands, aardal@cs.ruu.nl \\ Martine Labbé \\ Service de Mathématiques de la Gestion, Université Libre de Bruxelles \\ Bruxelles, Belgium, mlabbe@ulb.ac.be \\ Janny Leung \\ Management Information Systems Department, University of Arizona \\ Tucson, Arizona, USA, jleung@ventana.bpa.arizona.edu \\ Maurice Queyranne \\ Faculty of Commerce and Business Administration, University of British Columbia \\ Vancouver, British Columbia, Canada, queyranne@mtsg.ubc.ca
}

\begin{abstract}
We study the two-level uncapacitated facility location (TUFL) problem. Given two types of facilities, which we call y-facilities and z-facilities, the problem is to decide which facilities of both types to open, and to which pair of y- and z-facilities each client should be assigned, in order to satisfy the demand at maximum profit.

We first present two multi-commodity flow formulations of TUFL and investigate the relationship between these formulations and similar formulations of the one-level uncapacitated facility location (UFL) problem. In particular, we show that all nontrivial facets for UFL define facets for the two-level problem, and derive conditions when facets of TUFL are also facets for UFL. For both formulations of TUFL, we introduce new families of facets and valid inequalities and discuss the associated separation problems. We also characterize the extreme points of the LP-relaxation of the first formulation.

While the LP-relaxation of a multi-commodity formulation provides good bounds in general, the number of variables and constraints grows rapidly with the size of the problem instance. An alternative model of TUFL is a single-commodity fixed-charge network flow problem. Rardin and Wolsey showed that by projecting a so-called multicommodity extended formulation of fixed-charge network flow problems onto the space of flow variables used in the weaker flow formulation, a broad class of valid inequalities can be obtained. We discuss a subclass of these inequalities for TUFL that seems particularly useful for computational purposes.
\end{abstract}

Subject classification: $\bar{F}$ acility Location: Discrete; Integer Programming: Cutting planes/Facets; Transportation: Location Models. 
The two-level uncapacitated facility location (TUFL) problem involves two types of facilities which we call y-facilities and z-facilities. Decisions have to be made simultaneously on which facilities of both types to open Tand to which pair of y-and z-facility each client should be assigned. The objective is to maximize profit under the constraint that the demand of all clients has to be met.

The two-level problem is a natural extension of the well-known uncapacitated facility location (UFL) problem. This two-level problem can be used to model a hierarchical structure with a set of major facilities connected to minor facilities $\Gamma$ which in turn are connected to clients. Many practical location situations have a clear two-level structure. For example $\Gamma$ the distribution networks of many companies often involve major (central) as well as minor (regional) depots. The central facilities supply the regional ones and such shipment quantities are typically large $\Gamma$ whereas each client is served from a regional depot where the transport is usually carried out using smaller vehicles. Other applications involve two types of non-hierarchical facilities. For exampleГin garbage collection $\Gamma$ a truck travels from a depot to the client and then to a disposal plant so each client is assigned to a truck depot and a disposal plant. In situations where spent products are recycled $\Gamma$ each client have to be assigned to a supply facility and a recycling facility. Numerous other applications of TUFL exist within areas such as telecommunication and computer network design. Some applications are described in Barros and Labbé (1992).

Very little is known about the structural properties of TUFLT except that it is an NP-hard problem

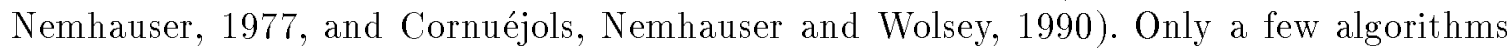
for TUFL have been developed. Kaufman et al. (1977) developed a branch-and-bound algorithm generalizing an algorithm for UFL developed by Efroymson and Ray (1966). Tcha and Lee (1984) also developed a branch-and-bound method where a dual ascent procedure $\Gamma$ similar to the one developed by Erlenkotter (1978) Гis used together with a primal descent method to get good lower and upper bounds. Barros and Labbé (1992) studied various formulations of a problem related to TUFLT and suggested a Lagrangean relaxation and a primal heuristic to derive bounds in a branch-and-bound algorithm. For the capacitated two-level problem Aardal (1992) studied the cutting plane approach. Balakrishnan and Graves (1989) consider a generalization of TUFL and develop algorithms for finding good lower and upper bounds on the optimal solution value.

We investigate some structural properties of TUFL. In Section $1 \Gamma$ we introduce the necessary notation and a multi-commodity flow formulation of TUFLTand characterize the extreme points of its LP-relaxation. We investigate the relationship between TUFL and UFL in Section 2. Since UFL is a relaxation of TUFLTany inequality valid for UFL is also valid for TUFL. We show that certain inequalities that define facets for TUFL induce facets for UFLTand that basically all facet-defining inequalities for UFL can be extended to facet-defining inequalities for TUFL. ConsequentlyГwe obtain several classes of facets for TUFL from previously-known facet-defining inequalities for UFL (see Cho et al. $1983 \mathrm{a} \mathrm{Ib} \Gamma$ 
Cornuéjols and ThizyГ1982ГGuignardГ1980Гand Cornuéjols et al.Г1977).

In Section 35we introduce two sets of constraints to the multi-commodity flow formulation which ensure that a facility is not open unless a client is assigned to it. We present new families of facets and valid inequalities for both formulationsTand discuss the corresponding separation problems. If we consider UFL with the extra constraints that a facility cannot be opened unless a client is assigned to it $\Gamma$ we show in Section 4 that almost all facets of this variant of UFL can be extended to facets for the corresponding version of TUFL.

In Section 5Twe present a single-commodity flow formulation of TUFL. This formulation provides a weaker LP-bound than the multi-commodity flow formulation Tbut may for large instances be computationally more tractable Tas it contains substantially fewer variables and constraints. In order to strengthen the flow formulation Twe consider a subclass of a general family of inequalities called dicut-collection inequalities $\Gamma$ developed by Rardin and Wolsey (1993). The general class is obtained by projecting a so-called multi-commodity extended formulation onto the space of the variables of the flow formulation.

\section{A Multi-Commodity Formulation of TUFL}

Here we introduce a formulation of the two-level uncapacitated facility location problem that is a natural extension of the one-level facility location problem. This formulation can be viewed as a multi-commodity fixed-charge network flow problem.

Let $I \Gamma J \Gamma$ and $K$ denote the sets of z-facilities $\Gamma \mathrm{y}$-facilities and clients respectively. Let $m=|I|, n=|J|$ and $q=|K|$. Furthermore $l$ let

$$
\begin{aligned}
z_{i} & = \begin{cases}1 & \text { if z-facility } i \text { is open } \Gamma \\
0 & \text { otherwise } \Gamma\end{cases} \\
y_{j} & = \begin{cases}1 & \text { if } \mathrm{y} \text {-facility } j \text { is open } \Gamma \\
0 & \text { otherwise } \Gamma \text { and }\end{cases} \\
x_{i j k} & =\text { fraction of demand of client } k \text { that is served by z-facility } i \text { and } \mathrm{y} \text {-facility } j .
\end{aligned}
$$

We want to determine which z- and y-facilities should be opened and to which pair of facilities each client should be assigned so as to maximize profit under the constraint that all demand has to be satisfied. To avoid trivial casesTwe henceforth assume that $m \Gamma n$ and $q$ are all at least equal to 2. 
The two-level facility location problem can be modeled as:

$(\mathrm{MC})$ :

$$
\max \sum_{i \in I} \sum_{j \in J} \sum_{k \in K} c_{i j k} x_{i j k}-\sum_{j \in J} f_{j} y_{j}-\sum_{i \in I} g_{i} z_{i}
$$

subject to

$$
\begin{array}{rll}
\sum_{i \in I} \sum_{j \in J} x_{i j k} & =1 & \text { for all } k \in K, \\
\sum_{j \in J} x_{i j k} & \leq z_{i} & \text { for all } i \in I, k \in K, \\
\sum_{i \in I} x_{i j k} & \leq y_{j} & \text { for all } j \in J, k \in K, \\
0 \leq z_{i} & \leq 1 & \text { for all } i \in I, \\
0 \leq y_{j} & \leq 1 & \text { for all } j \in J, \\
x_{i j k} & \geq 0 & \text { for all } i \in I, j \in J, k \in K, \\
z_{i}, y_{j} \text { integer } & \text { valued } & \text { for all } i \in I, j \in J,
\end{array}
$$

where $c_{i j k}$ is the profit of supplying the demand of client $k$ from z-facility $i$ and $y$-facility $j \Gamma f_{j}$ and $g_{i}$ are the fixed costs of opening $\mathrm{y}$-facility $j$ and $\mathrm{z}$-facility $i$ respectively.

Model MC is sufficient even for the case when the demand of each client is not allowed to be split. Because there are no capacity constraints on the depots and facilitiesTthe problem will always have an optimal solution where the $x_{i j k}$ 's are integer-valued. Let

$$
\begin{aligned}
& X_{\mathrm{MC}}=\left\{(x, y, z) \in R^{m n q+m+n}:(x, y, z) \text { satisfies }(1)-(7)\right\}, \\
& \bar{X}_{\mathrm{MC}}=\left\{(x, y, z) \in R^{m n q+m+n}:(x, y, z) \text { satisfies }(1)-(6)\right\},
\end{aligned}
$$

and let $P_{\mathrm{MC}}=\operatorname{conv}\left(X_{\mathrm{MC}}\right)$. An alternative model of TUFL is obtained by considering each pair $(i, j)$ of $z$ - and $\mathrm{y}$-facilities as a single uncapacitated facility represented by the 0 -1variable $w_{i j}$ Tthus obtaining an UFL-type model. The fixed charges are correctly accounted for by introducing variables $z_{i}, i \in I$ Tand $y_{j}, j \in J$ Tand the forcing constraints

$$
w_{i j} \leq z_{i} \text {, and } w_{i j} \leq y_{j} \text {, for all } i \in I, j \in J \text {. }
$$

This model allows the results for UFL to be applied directly an additional $m \times n$ 0-1-variables.

Below we derive the dimension of $P_{\mathrm{MC}}$ and determine which of the defining inequalities are facet defining. The proofs of Propositions 1 and 2 (part $c$ ) are straightforward and are omitted. We defer the proofs of the rest of Proposition 2 to Section 2.

Proposition 1 The dimension of the polytope $P_{\mathrm{MC}}$ is $m n q+m+n-q$. 


\section{Proposition 2}

(a) For any $j \in J$ and $k \in K, \sum_{i \in I} x_{i j k} \leq y_{j}$ defines a facet of $P_{\mathrm{MC}}$.

(b) For any $i \in I$ and $k \in K, \sum_{j \in J} x_{i j k} \leq z_{i}$ defines a facet of $P_{\mathrm{MC}}$.

(c) For any $i \in I, j \in J$ and $k \in K, x_{i j k} \geq 0$ defines a facet of $P_{\mathrm{MC}}$.

(d) For any $j \in J, y_{j} \leq 1$ defines a facet of $P_{\mathrm{MC}}$.

(e) For any $i \in I, z_{i} \leq 1$ defines a facet of $P_{\mathrm{MC}}$.

\subsection{Extreme Points of $\bar{X}_{\mathrm{MC}}$}

The next proposition gives a characterization of the extreme points of the LP-relaxation $\bar{X}_{\mathrm{MC}}$. Let $(x, y, z)$ be a point in $\bar{X}_{\mathrm{MC}}$. We define the sets

$$
\begin{aligned}
I_{1} & =\left\{i: 0<z_{i}<1\right\} \\
J_{1} & =\left\{j: 0<y_{j}<1\right\}, \text { and } \\
K_{1} & =\left\{k: \text { there exists an }(i, j) \text { pair such that } 0<x_{i j k}<1\right\} .
\end{aligned}
$$

For each $k \in K_{1}$ Twe define the sets

$$
\begin{aligned}
\tilde{I}_{k} & =\left\{i: 0<\sum_{j \in J} x_{i j k} \leq z_{i}<1 \text { or } 0<\sum_{j \in J} x_{i j k}<z_{i}=1\right\}, \\
\text { and } \tilde{J}_{k} & =\left\{j: 0<\sum_{i \in I} x_{i j k} \leq y_{j}<1 \text { or } 0<\sum_{i \in I} x_{i j k}<y_{j}=1\right\} .
\end{aligned}
$$

and the directed graph $G_{k}=\left(N_{k}, A_{k}\right)$ where $N_{k}=\{s, t, d\} \cup \tilde{I}_{k} \cup \tilde{J}_{k}$ and

$$
\begin{aligned}
A_{k}= & \left\{(s, i): i \in \tilde{I}_{k} \cap I_{1}\right\} \\
& \cup\left\{(i, j): x_{i j k}>0, i \in \tilde{I}_{k}, j \in \tilde{J}_{k}\right\} \\
& \cup\left\{(j, t): j \in \tilde{J}_{k} \cap J_{1}\right\} \\
& \cup\left\{(d, i): \sum_{j \in J} x_{i j k}<z_{i}, i \in \tilde{I}_{k}\right\} \\
& \cup\left\{(j, d): \sum_{i \in I} x_{i j k}<y_{j}, j \in \tilde{J}_{k}\right\} .
\end{aligned}
$$

The arcs incident to the dummy node $d$ indicate which of the constraints (2) and (3) are slack for client $k$. An arc incident to the dummy source node $s$ indicates that the corresponding $z$-variable is fractional; similarly the arcs $(j, t)$ indicate that $y_{j}$ is fractional. 


\section{Example 1}

Consider the following point in $\bar{X}_{\mathrm{MC}}$ for $I=J=\{1,2,3\}$ and $K=\{1,2\}$ :

$$
\begin{aligned}
& z_{1}=z_{2}=\frac{1}{3}, z_{3}=1, \quad y_{1}=y_{2}=\frac{1}{3}, y_{3}=1, \\
& x_{131}=x_{211}=x_{321}=\frac{1}{3}, \quad x_{122}=x_{232}=x_{312}=\frac{1}{3}, \quad \text { all other } x_{i j k}=0 .
\end{aligned}
$$

For this point

$$
I_{1}=J_{1}=\{1,2\}, \quad \tilde{I}_{1}=\tilde{J}_{1}=\{1,2,3\}, \quad \tilde{I}_{2}=\tilde{J}_{2}=\{1,2,3\},
$$

and the graphs $G_{1}$ and $G_{2}$ are shown in Figure 1.

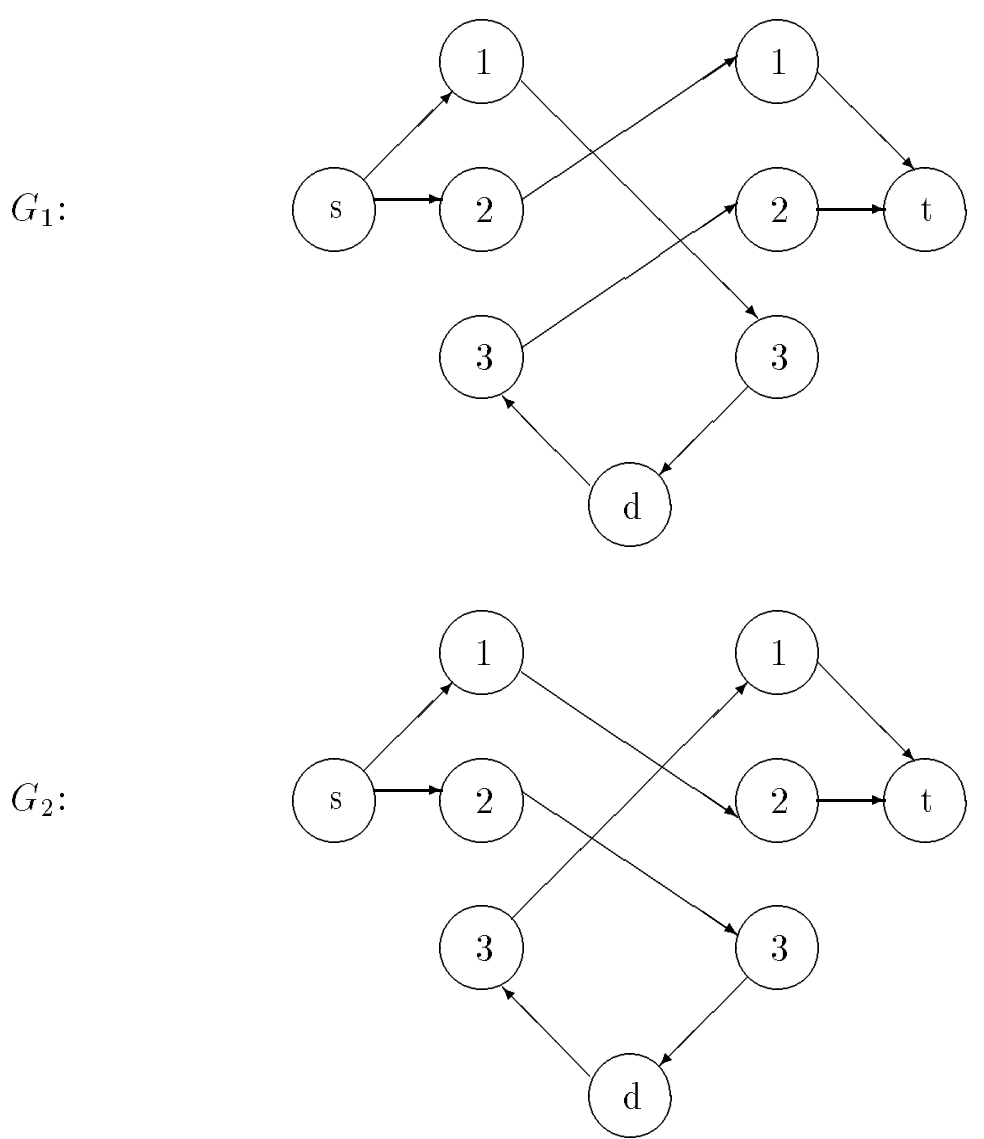

Figure 1: The graphs $G_{1}$ and $G_{2}$ for Example 1. 
We call $f \in \mathbf{R}^{A_{k}}$ a pseudo-feasible flow for $G_{k}$ if flow balance is maintained at each node in $G_{k}$ except node $d$; that is $\Gamma$ for each node $v \in N_{k} \backslash\{d\} \Gamma$

$$
\sum_{u \in N_{k}:(u, v) \in A_{k}} f_{(u, v)}=\sum_{w \in N_{k}:(v, w) \in A_{k}} f_{(v, w)} .
$$

Note that we allow the flow value for an arc to be either positive or negative. Let $\hat{G}_{k}$ be the subgraph of $G_{k}$ induced by the node-set $\tilde{I}_{k} \cup \tilde{J}_{k} \cup\{d\}$.

Theorem $3(x, y, z) \in \bar{X}_{\mathrm{MC}}$ is an extreme point of $\bar{X}_{\mathrm{MC}}$ if and only if

(a) for all $i \in I_{1}, z_{i}=\max _{k} \sum_{j \in J} x_{i j k}$,

(b) for all $j \in J_{1}, y_{j}=\max _{k} \sum_{i \in I} x_{i j k}$,

(c) for any $k$ in $K_{1}$, the graph $\hat{G}_{k}$ does not contain any even undirected circuit,

(d) there is no non-zero vector $\left(\alpha^{\prime}, \beta^{\prime}\right) \in \mathbf{R}^{I_{1}} \times \mathbf{R}^{J_{1}}$ such that:

$$
\begin{aligned}
& \text { for each } k \in K_{1} \text {, there is a pseudo-feasible flow } f^{k} \text { for } G_{k} \text { with } f_{(s, i)}^{k}= \\
& \alpha_{i}^{\prime} \text { for all } i \in \tilde{I_{k}} \cap I_{1} \text { and } f_{(j, t)}^{k}=\beta_{j}^{\prime} \text { for all } j \in \tilde{J}_{k} \cap J_{1} \text {. }
\end{aligned}
$$

The vector $\left(\alpha^{\prime}, \beta^{\prime}\right)$ specifies a set of flow-values for the arcs incident to the source node $s$ and sink node $t$ that is held fixed for all the graphs $G_{k}, k \in K_{1}$. Condition (8) says that for each $k \in K_{1}$ Twe can assign flow-values to the remaining arcs in $G_{k}$ to give a pseudo-feasible flow.

This result generalizes an analogous result for the uncapacitated facility location problem in Cornuéjols FFisher and Nemhauser (1977). Since the proof is quite lengthy it is deferred to the Appendix.

\section{Adapting Facets from the One-Level Uncapacitated Facil- ity Location Problem}

The set of feasible solutions to the uncapacitated facility location problem (UFL) is defined as:

$$
\begin{aligned}
& X_{\mathrm{UFL}}=\left\{(x, y) \in R^{n q+n}: \sum_{j \in J} x_{j k} \quad=1 \quad \text { for all } k \in K,\right. \\
& x_{j k} \leq y_{j} \quad \text { for all } j \in J, k \in K, \\
& x_{j k} \quad \geq 0 \quad \text { for all } j \in J, k \in K \text {, }
\end{aligned}
$$




$$
\begin{gathered}
0 \leq y_{j} \quad \leq 1 \quad \text { for all } j \in J, \\
y_{j} \text { integer valued }
\end{gathered}
$$

where $x_{j k}$ is the fraction of client $k$ 's demand served by facility $j \Gamma$ and where $y_{j}=1$ if facility $j$ is open $\Gamma$ and $y_{j}=0$ otherwise. As before $\Gamma$ we assume that $n$ and $q$ are both at least equal to 2 . Let $P_{\mathrm{UFL}}=\operatorname{conv}\left(X_{\mathrm{UFL}}\right)$.

Proposition 4 (CornuéjolsTNemhauser and WolseyГ1990) The dimension of $P_{\mathrm{UFL}}$ is $n q+n-q$.

Below we show that all nontrivial facets of UFL are also facets for $P_{\mathrm{MC}}$ Tthus providing us with a large collection of facets for TUFL.

\section{Theorem 5 Let}

$$
\sum_{j \in J} \sum_{k \in K} a_{j k} x_{j k}+\sum_{j \in J} b_{j} y_{j} \leq d
$$

represent a facet of $P_{\mathrm{UFL}}$ that is not a non-negativity constraint for some $x_{j k}$. Then

$$
\sum_{j \in J} \sum_{k \in K} a_{j k} \sum_{i \in I} x_{i j k}+\sum_{j \in J} b_{j} y_{j} \leq d
$$

represents a facet of $P_{\mathrm{MC}}$.

Proof. $\quad$ Let $\mathcal{F} \equiv\left\{(x, y, z) \in P_{\mathrm{MC}}: \sum_{j \in J} \sum_{k \in K} a_{j k} \sum_{i \in I} x_{i j k}+\sum_{j \in J} b_{j} y_{j}=d\right\} \Gamma$ and let $\alpha x+\beta y+$ $\gamma z \leq \delta$ be a valid inequality for $P_{\mathrm{MC}}$ such that for all $(x, y, z) \in \mathcal{F} \Gamma$

$$
\sum_{i \in I} \sum_{j \in J} \sum_{k \in K} \alpha_{i j k} x_{i j k}+\sum_{j \in J} \beta_{j} y_{j}+\sum_{i \in I} \gamma_{i} z_{i}=\delta
$$

Let $\mathcal{F}_{1} \equiv\left\{(x, y) \in P_{\mathrm{UFL}}: \sum_{j \in J} \sum_{k \in K} a_{j k} x_{j k}+\sum_{j \in J} b_{j} y_{j}=d\right\}$. We will show that there exists $\theta \in \mathbb{R}^{1}$ and $\eta_{k} \in \mathbb{R}^{1}, k=1, \ldots, q$ such that

$$
\begin{aligned}
\alpha_{i j k} & =\theta a_{j k}+\eta_{k} & & \text { for all } i \in I, j \in J, k \in K, \\
\beta_{j} & =\theta b_{j} & & \text { for all } j \in J, \\
\gamma_{i} & =0 & & \text { for all } i \in I, \\
\delta & =\theta d+\sum_{k \in K} \eta_{k} . & &
\end{aligned}
$$

By Theorem 3.6 in Section I.4 of Nemhauser and Wolsey (1988) Tthis would imply that the inequality $\alpha x+\beta y+\gamma z \leq \delta$ defines a facet of $P_{\mathrm{MC}}$.

First $\Gamma$ let $(x, y)$ be a point in $\mathcal{F}_{1}$. From this point $\Gamma$ we can construct two points $(\bar{x}, y, \bar{z}) \Gamma$ $(\bar{x}, y, \tilde{z}) \in \mathcal{F}$ as follows: 
- $\bar{z}_{1}=1, \bar{z}_{i}=0$ for $i \neq 1 \Gamma$

- $\tilde{z}_{1}=1, \tilde{z}_{2}=1, \tilde{z}_{i}=0$ for $i \neq 1,2 \Gamma$

- the $y_{j}$-values are unchanged for all $j \in J \Gamma$

- for all $j \in J$ and $k \in K \Gamma \bar{x}_{1 j k}=x_{j k}, \bar{x}_{i j k}=0$ for $i \neq 1$.

These two points correspond to allocating all clients to z-facility 1 . For the first point Tonly z-facility 1 is open; both $z$-facilities 1 and 2 are open for the second point. By evaluating (10) at the above points we obtain

$$
\begin{aligned}
& \sum_{j \in J} \sum_{k \in K} \alpha_{1 j k} x_{j k}+\sum_{j \in J} \beta_{j} y_{j}+\gamma_{1}=\delta, \\
& \sum_{j \in J} \sum_{k \in K} \alpha_{1 j k} x_{j k}+\sum_{j \in J} \beta_{j} y_{j}+\gamma_{1}+\gamma_{2}=\delta .
\end{aligned}
$$

Hence $\Gamma \gamma_{2}=0$ Tand by symmetry $\Gamma \gamma_{i}=0$ for all $i \in I$.

From now on $\Gamma$ all the points $(x, y, z) \in \mathcal{F}$ that we consider are such that $z_{i}=1 \Gamma$ for all $i$. Since we assume that $(9)$ is not a nonnegativity constraint then $\Gamma$ for any y-facility $j^{\prime}$ and any client $k^{\prime}$ Tthere is a point in $\mathcal{F}_{1}$ with $x_{j^{\prime} k^{\prime}}=1$. For simplicity with $x_{11}=1$. From this point we can construct $m$ points $\Gamma\left(x^{l}, y, 1\right) \in \mathcal{F} \Gamma$ for $l=1, \ldots, m \Gamma$ as follows:

- the $y_{j}$-values are unchanged for all $j \in J \Gamma$

- $x_{i 11}^{l}=\left\{\begin{array}{ll}1 & \text { if } i=l \Gamma \\ 0 & \text { otherwise } \Gamma\end{array} \quad\right.$ for all $i \in I$,

$$
\begin{array}{ll}
x_{i j 1}^{l}=0, & \text { for all } i \in I, j \in J, j \neq 1, \\
x_{i j k}^{l}=\left\{\begin{array}{ll}
x_{j k} & \text { if } i=m \Gamma \\
0 & \text { otherwise }
\end{array} \text { for all } j \in J, k \in K, k \neq 1 .\right.
\end{array}
$$

In the $l$-th point $\Gamma$ client 1 is allocated to $y$-facility 1 and $z$-facility $l$. All other clients are allocated to the same $\mathrm{y}$-facilities as indicated by $(x, y)$ Tand to the last $z$-facility $m$. By evaluating (10) for $\left(x^{1}, y, 1\right),\left(x^{2}, y, 1\right), \ldots,\left(x^{m}, y, 1\right)$ we obtain for $l=1, \ldots, m$ :

$$
\alpha_{l 11}+\sum_{j \in J} \sum_{\substack{c \in K \\ k \neq 1}} \alpha_{m j k} x_{j k}+\sum_{j \in J} \beta_{j} y_{j}=\delta .
$$

Hence $\Gamma \alpha_{l 11}$ is equal to some constant $\alpha_{11}$ for $l=1, \ldots, m$. By symmetryГwe obtain that for all $j \in J$ and $k \in K \Gamma \alpha_{i j k}=\alpha_{j k}$ for all $i \in I$.

Finallyएlet $(x, y)$ be any point in $\mathcal{F}_{1}$ and construct the point $(x, y, 1) \in \mathcal{F}$ as follows: 
- the $y_{j}$-values are unchanged for all $j \in J \Gamma$

- $x_{1 j k}=x_{j k}, \quad$ for all $j i n J, k \in K$,

$x_{i j k}=0, \quad$ for all $i \in I, i \neq 1, j \in J, k \in K$.

Here $\Gamma$ all clients are assigned to z-facility 1 . Evaluating $(10)$ at $(x, y, 1)$ gives

$$
\sum_{j \in J} \sum_{k \in K} \alpha_{j k} x_{j k}+\sum_{j \in J} \beta_{j} y_{j}=\delta .
$$

Since this holds for any point in $\mathcal{F}_{1}$ and since $\mathcal{F}_{1}$ is a facet of $P_{\mathrm{UFL}} \Gamma$ we know that there exists $\theta \in \mathbb{R}^{1}$ with $\theta>0$ Tand $\eta_{k} \in \mathbb{R}^{1}$ such that

$$
\begin{array}{rlr}
\alpha_{j k} & =\theta a_{j k}+\eta_{k} \quad & \text { for all } j \in J, k \in K, \\
\beta_{j} & =\theta b_{j} & \text { for all } j \in J, \\
\delta & =\theta d+\sum_{k \in K} \eta_{k}, &
\end{array}
$$

which completes the proof.

Next Twe show that some facets of the polytope for the two-level problem also induce facets for the one-level problem. More precisely $\mathrm{C}$ a facet of $P_{\mathrm{MC}}$ is also facet-defining for $P_{\mathrm{UFL}}$ as long as the coefficients of all $z_{i}$-variables are equal to zero $\Gamma$ and as long as the coefficients of the variables $x_{i j k}$ are independent of $i$.

Theorem 6 Every facet of $P_{\mathrm{MC}}$ of the form

$$
\sum_{j \in J} \sum_{k \in K} a_{j k} \sum_{i \in I} x_{i j k}+\sum_{j \in J} b_{j} y_{j} \leq d,
$$

where the coefficient of $z_{i}$ is zero, for all $i \in I$, and the coefficient of $x_{i j k}$ is independent of $i$ for all $i \in I, j \in J, k \in K$, induces a facet for $P_{\mathrm{UFL}}$.

Proof. Let $p$ denote the dimension of $P_{\mathrm{MC}}$. If (11) represents a facet of $P_{\mathrm{MC}}$ Tthen we can construct a $p \times(m n q+m+n+1)$ matrix of rank $p$ of the form $[\mathbf{M} \mid \mathbf{1}]$ where the elements in the last column are all 1'sTand each row of $\mathrm{M}$ is the vector defining a point in $P_{\mathrm{MC}}$ lying on the facet represented by (11). Since the rank of a matrix is unchanged by column operations $\Gamma$ the rank of this matrix is unchanged if $\Gamma$ for each $j$ and each $k \Gamma$ the column corresponding to the variable $x_{1 j k}$ is replaced by the sum of the columns corresponding to $x_{i j k}$ for $i=1, \ldots, m$.

Now consider a submatrix $\left[\mathbf{M}^{\prime} \mid \mathbf{1}\right]$ obtained by deleting the columns corresponding to $z_{i}$ for $i=1, \ldots, m$ and $x_{i j k}$ for $i=2, \ldots, m, j=1, \ldots, n$, and $k=1, \ldots, q$. The rank of this matrix is at least $p-(n q(m-1)+m)=n q+n-q$ since $p=m n q+m+n-q$. Each row of this submatrix corresponds to a point in $X_{\mathrm{UFL}}$ on the facet defined by (9). 
Since the inequalities

$$
\text { and } \quad \begin{aligned}
x_{j k} \leq y_{j} & \text { for all } j \in J, k \in K, \\
y_{j} \leq 1 & \text { for all } j \in J,
\end{aligned}
$$

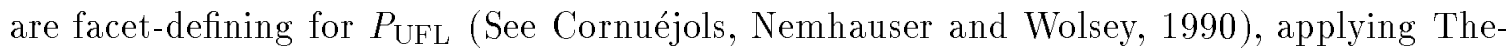
orem 5 proves parts $(\mathrm{a})$ and $(\mathrm{d})$ of Proposition 2. Furthermore $\mathrm{since}$ the roles of the $\mathrm{z}$ and the $y$-facilities are symmetric Tan analogous collection of facets can be derived by interchanging the roles of the indices $i$ and $j$ Tand correspondingly the variables $z_{i}$ and $y_{j}$ Tthus proving parts (b) and (e) of Proposition 2.

In addition to identifying the trivial facets of $P_{\mathrm{MC}}$ T Theorem 5 can be exploited more generally to yield many more facet-defining inequalities for $P_{\mathrm{MC}}$. Cornuéjols et al. (1977) $\Gamma$ Guignard (1980)ГCornuéjols and Thizy (1982) introduced several classes of facets for $P_{\mathrm{UFL}}$. Later $\Gamma$ Cho et al. $(1983 \mathrm{aIb})$ developed a general class of facet-defining inequalities that subsumes all previously-known facets of $P_{\mathrm{UFL}}$. By applying Theorem 5 to these results Twe obtain a large collection of facets for $P_{\mathrm{MC}}$ involving the clients and the $\mathrm{y}$-facilitiesTand an analogous collection of facets for $P_{\mathrm{MC}}$ involving the clients and the $z$-facilities.

\section{New Facets for TUFL}

Here we introduce two new classes of facet-defining inequalities for TUFL. We first describe in Section 3.1Tfacets of $P_{\mathrm{MC}} \Gamma$ and introduce $\Gamma$ in Section $3.2 \Gamma$ facets of a slightly modified polytope. Both classes involve either $z$ - and $x$-variables or $y$ - and $x$-variables. Since the roles of z- and $y$-facilities are interchangeable $\Gamma$ such classes of facets always come in pairs.

\subsection{New Facets of $P_{\mathrm{MC}}$}

\subsubsection{The Valid Inequalities}

Consider $p$ clients $\mathrm{T}$ and assume that precisely one of the $p$ clients is assigned to every $\mathrm{y}$ facility. If only one $\mathrm{y}$-facility $\Gamma$ say $j_{1}$ is open $\Gamma$ then all clientsTincluding the designated client $k\left(j_{1}\right) \Gamma$ have to be assigned to facility $j_{1} \Gamma$ since all demand must be met $\Gamma$ leading to the following valid inequality.

Proposition 7 Assume that $q \geq n$ and let $S=\{k(1), k(2), \ldots, k(n)\} \subseteq K$ be any sequence of $n$ distinct clients. Then

$$
\sum_{j \in J}\left(y_{j}+\sum_{i \in I} x_{i j k(j)}\right) \geq 2
$$

is valid for $X_{\mathrm{MC}}$. 
Theorem 8 If $q \geq n \geq 3$, then inequality (12) defines a facet of $P_{\mathrm{MC}}$.

Proof. We will prove that (12) represents a facet using the indirect method. Let $\alpha x+$ $\beta y+\gamma z \leq \delta$ be valid for $P_{\mathrm{MC}}$ Tand assume that

$$
\begin{aligned}
\mathcal{F} & \equiv\left\{(x, y, z) \in P_{\mathrm{MC}}: \sum_{j \in J}\left(y_{j}+\sum_{i \in I} x_{i j k(j)}\right)=2\right\} \\
& \subseteq\left\{(x, y, z) \in P_{\mathrm{MC}}: \sum_{i \in I} \sum_{j \in J} \sum_{k \in K} \alpha_{i j k} x_{i j k}+\sum_{j \in J} \beta_{j} y_{j}+\sum_{i \in I} \gamma_{i} z_{i}=\delta\right\} .
\end{aligned}
$$

To prove that inequality (12) defines facets of $P_{\mathrm{MC}}$ when $q \geq n \geq 3$ Twe need to show that

$$
\begin{aligned}
\alpha_{i j k} & =-\theta+\eta_{k} & & \text { for all } i \in I, j \in J, k(j) \in S, \\
\alpha_{i j k} & =\eta_{k} & & \text { for all } i \in I, j \in J, k \in K, k \neq k(j), \\
\beta_{j} & =-\theta & & \text { for all } j \in J, \\
\gamma_{i} & =0 & & \text { for all } i \in I, \\
\delta & =-2 \theta+\sum_{k \in K} \eta_{k} & &
\end{aligned}
$$

for some constants $\theta>0$ and $\eta_{k}, k \in K$.

Choose arbitrarily one z-facility $i_{1}$ Ttwo y-facilities $j_{1}, j_{2}$ and consider the following tight point in $\mathcal{F}$ with:

$$
\begin{aligned}
& \begin{aligned}
z_{i_{1}} & =1 \\
y_{j_{1}}=y_{j_{2}} & =1
\end{aligned} \\
& x_{i_{1}, j_{1} k\left(j_{2}\right)}=1 \\
& \begin{aligned}
x_{i_{1} j_{2} k\left(j_{1}\right)} & =1 \\
x_{i_{1} j_{1} k} & =1
\end{aligned} \quad \text { for all } k \in K, k \neq k\left(j_{1}\right) \text { or } k\left(j_{2}\right) \text {, }
\end{aligned}
$$

and all other elements with value zero. We refer to the above point as point 1 . Consider a second point with $z_{i_{2}}=1, i_{2} \neq i_{1}$ Tand other elements as in point 1 . By evaluating the expression

$$
\sum_{i \in I} \sum_{j \in J} \sum_{k \in K} \alpha_{i j k} x_{i j k}+\sum_{j \in J} \beta_{j} y_{j}+\sum_{i \in I} \gamma_{i} z_{i}=\delta
$$

at these two points $\Gamma$ we obtain $\gamma_{i_{2}}=0$. Varying over all possible choices of $i_{2}$ gives $\gamma_{i}=0$ for all $i \in I, i \neq i_{1}$ and since $i_{1}$ was chosen arbitrarily we get

$$
\gamma_{i}=0 \text { for all } i \in I \text {. }
$$


Next $\Gamma$ consider the modification of point 1 where $z_{i_{2}}=1$ as well for $i_{2} \neq i_{1}$ Tand where one arbitrarily chosen client $k_{1}$ is assigned to z-facility $i_{2}$. Evaluating (13) at this point and at point 1 gives $\alpha_{i_{1} j_{1} k_{1}}=\alpha_{i_{2} j_{1} k_{1}}$. Since $i_{1}, j_{1}$ and $k_{1}$ were chosen arbitrarily we have

$$
\alpha_{i j k}=\alpha_{j k} \text {, for all } i \in I, j \in J, k \in K \text {. }
$$

We now consider point 1 and choose an arbitrary client $k_{1}$ Гdifferent from clients $k\left(j_{1}\right), k\left(j_{2}\right) \Gamma$ and we re-assign client $k_{1}$ to $y$-facility $j_{2}$. Evaluate (13) at the new point and at point 1 Tand compare the expressions. This gives $\alpha_{j_{1} k_{1}}=\alpha_{j_{2} k_{1}}$. Knowing that $j_{1}$ and $j_{2}$ were arbitrarily chosen and that $k_{1}$ is any client different from client $k\left(j_{1}\right)$ and $k\left(j_{2}\right)$ yields

$$
\alpha_{j k}=\eta_{k} \text { for all } j \in J, k \in K, k \neq k(j) .
$$

Next consider a point in $\mathcal{F}$ with the same structure as point $1 \Gamma$ but where a y-facility $j_{3} \neq j_{1}$ or $j_{2}$ takes the role of $\mathrm{y}$-facility $j_{2}$ Ti.e.

$$
\begin{aligned}
& \begin{aligned}
z_{i_{1}} & =1 \\
y_{j_{1}}=y_{j_{3}} & =1
\end{aligned} \\
& x_{i_{1} j_{1} k\left(j_{3}\right)}=1 \\
& x_{i_{1} j_{3} k\left(j_{1}\right)}=1 \\
& x_{i_{1} j_{1} k}=1 \quad \text { for all } k \in K, k \neq k\left(j_{1}\right) \text { or } k\left(j_{3}\right) \text {, }
\end{aligned}
$$

and all other variables are equal to zero. By evaluating (13) at the above point and at point $1 \Gamma$ we get $\alpha_{j_{2} k\left(j_{1}\right)}-\alpha_{j_{3} k\left(j_{1}\right)}+\beta_{j_{2}}-\beta_{j_{3}}=0$. Since $\alpha_{j_{2} k\left(j_{1}\right)}=\alpha_{j_{3} k\left(j_{1}\right)}=\eta_{k\left(j_{1}\right)}$ we have $\beta_{j_{2}}=\beta_{j_{3}}$ Tand as $j_{2}$ and $j_{3}$ can be chosen arbitrarily we obtain

$$
\beta_{j}=-\theta \text { for all } j \in J,
$$

and since (12) is not an improper face we must have $\theta>0$.

Finally consider the following point in $\mathcal{F}$ that we refer to as point 2. Choose $i_{1}$ and $j_{1}$ as in point 1 Tand let

$$
\begin{aligned}
z_{i_{1}} & =1 \\
y_{j_{1}} & =1 \\
x_{i_{1} j_{1} k\left(j_{1}\right)} & =1 \\
x_{i_{1} j_{1} k\left(j_{2}\right)} & =1 \\
x_{i_{1} j_{1} k} & =1 \quad \text { for all } k \in K, k \neq k\left(j_{1}\right) \text { or } k\left(j_{2}\right) .
\end{aligned}
$$

Comparing point 1 to point 2 and using (14) Tand (15) gives $\alpha_{j_{1} k\left(j_{1}\right)}=-\theta+\eta_{k\left(j_{1}\right)}$. Given that $j_{1}$ is an arbitrary $y$-facility we obtain

$$
\alpha_{j k}=-\theta+\eta_{k(j)} \text { for all } j \in J, \text { and } k(j) \in S .
$$


Evaluating (13) at any point in $\mathcal{F}$ gives

$$
\delta=-2 \theta+\sum_{k \in K} \eta_{k},
$$

which concludes our proof.

Corollary 9 Assume that $q \geq m$ and let $S=\{k(1), k(2), \ldots, k(n)\} \subseteq K$ be any sequence of $n$ distinct clients. Then inequality

$$
\sum_{i \in I}\left(z_{i}+\sum_{j \in J} x_{i j k(j)}\right) \geq 2
$$

is valid for $X_{\mathrm{MC}}$ and defines a facet of $P_{\mathrm{MC}}$ if $q \geq m \geq 3$.

\subsubsection{Separation}

Inequality (12)Tand similarly (16)Гcan be separated by solving a minimum-weight maximumcardinality matching problem. For any point $(\bar{x}, \bar{y}, \bar{z}) \Gamma$ we consider the complete bipartite graph $G=(J \cup K, J \times K)$ with edge-weights

$$
w_{j k}=\bar{y}_{j}+\sum_{i \in I} \bar{x}_{i j k} \quad \forall(j, k) \in J \times K .
$$

Let the minimum-weight matching among all matchings of cardinality $n$ be

$$
M=\{(1, k(1)),(2, k(2)), \ldots,(n, k(n))\} .
$$

If the total weight of the matching $M$ is less than $2 \Gamma$ then inequality (12) Twith the sequence $S$ as defined by the edges of the matching $M$ above ${ }^{2}$ is a valid inequality that cuts off the point $(\bar{x}, \bar{y}, \bar{z})$.

\subsection{New Facets for a Modified Formulation}

The formulation MC allows a facility to be open even when no clients are assigned to it $\Gamma$ whereas in many applications we may require that a facility be closed when not used. When all the fixed costs are positive Tthe objective function ensures that this will be the case. For a more general cost structure $\Gamma$ it may be necessary to explicitly model this requirement $\Gamma$ thereby obtaining a different formulation of TUFL which we shall refer to as formulation $\mathrm{MC}^{\prime}$. 
Formulation $\mathrm{MC}^{\prime}$ is obtained by adding the following constraints to formulation $\mathrm{MC}$ :

$$
\begin{aligned}
& \sum_{j \in J} \sum_{k \in K} x_{i j k} \geq z_{i} \quad \text { for all } i \in I, \\
& \sum_{i \in I} \sum_{k \in K} x_{i j k} \geq y_{j} \text { for all } j \in J .
\end{aligned}
$$

Constraints (17) and (18) ensure that no facility is opened if no client is assigned to it. For inequalities (17) and (18) to be valid we need to explicitly state that the demand should not be split between clients. In formulation $\mathrm{MC}^{\prime}$ we therefore replace constraint (7) by

$$
x_{i j k}, y_{j}, z_{i} \text { integer valued for all } i \in I, j \in J, k \in K .
$$

Let

$$
X_{\mathrm{MC}^{\prime}}=\left\{(x, y, z) \in R^{m n q+m+n}:(x, y, z) \text { satisfies }(1)-(6),\left(7^{\prime}\right),(17)-(18)\right\} .
$$

Analogous to the the definitions of $\bar{X}_{\mathrm{MC}}$ and $P_{\mathrm{MC}}$ we define $\bar{X}_{\mathrm{MC}^{\prime}}$ and $P_{\mathrm{MC}}$ as

$$
\bar{X}_{\mathrm{MC}^{\prime}}=\left\{(x, y, z) \in R^{m n q+m+n}:(x, y, z) \text { satisfies }(1)-(6),(17)-(18)\right\} .
$$

and

$$
P_{\mathrm{MC}^{\prime}}=\operatorname{conv}\left(X_{\mathrm{MC}^{\prime}}\right) \text {. }
$$

\subsubsection{Dimension and Trivial Facets of $P_{\mathrm{MC}^{\prime}}$}

Here $\Gamma$ we state the results on the dimension and trivial facets for $P_{\mathrm{MC}^{\prime}}$. Since the proof techniques used for these results are standard they are omitted here.

Proposition 10 The dimension of the polytope $P_{\mathrm{MC}}$ is

(a) $m n q+n+n-q-2$ if $m=n=q=2$,

(b) $m n q+m+n-q-1$ if $q=2$ and either $m \geq 3, n=2$ or $m=2, n \geq 3$,

(c) $m n q+m+n-q$ in all other cases.

In the cases when $P_{\mathrm{MC}}$ is not full-dimensional $\Gamma$ all points in $P_{\mathrm{MC}}$ satisfy the following equations in addition to the demand constraints (1):

$$
\begin{array}{ll}
\text { If } n=2 \text { and } q=2: & y_{1}-\sum_{i \in I} \sum_{k \in K} x_{i 1 k}=y_{2}-1 . \\
\text { If } m=2 \text { and } q=2: & z_{1}-\sum_{j \in J} \sum_{k \in K} x_{1 j k}=z_{2}-1 .
\end{array}
$$


The trivial facets of parts (a) $\Gamma(\mathrm{b})$ and (c) of Proposition 2 are also facet defining with respect to $P_{\mathrm{MC}}$. In addition the following holds:

\section{Proposition 11}

(a) If $q \geq 3, z_{i} \leq 1$ represents a facet of $P_{\mathrm{MC}^{\prime}}$ for all $i \in I$.

(b) If $q \geq 3, y_{j} \leq 1$ represents a facet of $P_{\mathrm{MC}^{\prime}}$ for all $j \in J$.

(c) For any $j \in J, \sum_{i \in I} \sum_{k \in K} x_{i j k} \geq y_{j}$ defines a facet of $P_{\mathrm{MC}^{\prime}}$ unless $n=2$ and $q \geq 3$.

(d) For any $i \in I, \sum_{j \in J} \sum_{k \in K} x_{i j k} \geq z_{i}$ defines a facet of $P_{\mathrm{MC}^{\prime}}$ unless $m=2$ and $q \geq 3$.

Note that none of the inequalities $x_{i j k} \leq 1 \Gamma y_{j} \geq 0$ and $z_{i} \geq 0$ represent facets of $P_{\mathrm{MC}^{\prime}}$ as they are implied by the above inequalities. Also note that $x_{i j k} \leq y_{j}$ and $x_{i j k} \leq z_{i}$ are never facet defining.

\subsubsection{The Valid Inequalities}

The new inequalities introduced below are designed to capture structural properties involving y-facilities and clients as well as z-facilities and clients.

Theorem 12 Let $J^{\prime} \subseteq J$ with $1 \leq\left|J^{\prime}\right|<q$.

$$
\sum_{j \in J^{\prime}} y_{j}-\sum_{j \in J^{\prime}} \sum_{i \in I} \sum_{k \in K} x_{i j k}+\left(q-\left|J^{\prime}\right|\right)\left(1-\sum_{j \notin J^{\prime}} y_{j}\right) \leq 0
$$

is valid for $P_{\mathrm{MC}}$ and represents a facet of $P_{\mathrm{MC}^{\prime}}$ if $\left|J^{\prime}\right|<\min \{n, q\}$.

Note that when $n=q=2$ and $\left|J^{\prime}\right|=1 \Gamma(21)$ represents an improper facet of $P_{\mathrm{MC}^{\prime}}$; that isTall points in $P_{\mathrm{MC}^{\prime}}$ satisfy (21) with equality.

Proof of validity:

Case 1: $\quad \sum_{j \notin J^{\prime}} y_{j}=0$.

$$
\sum_{j \in J^{\prime}} y_{j}-\sum_{j \in J^{\prime}} \sum_{i \in I} \sum_{k \in K} x_{i j k}+\left(q-\left|J^{\prime}\right|\right)=\sum_{j \in J^{\prime}} y_{j}-\left|J^{\prime}\right| \leq 0,
$$

where the equality follows from $\sum_{j \in J^{\prime}} \sum_{i \in I} \sum_{k \in K} x_{i j k}=q \Gamma$ which holds since all demand has to be met. 
Case 2: $\quad \sum_{j \notin J^{\prime}} y_{j} \geq 1$.

$$
\sum_{j \in J^{\prime}} y_{j}-\sum_{j \in J^{\prime}} \sum_{i \in I} \sum_{k \in K} x_{i j k}+\left(q-\left|J^{\prime}\right|\right)\left(1-\sum_{j \notin J^{\prime}} y_{j}\right) \leq\left(q-\left|J^{\prime}\right|\right)\left(1-\sum_{j \notin J^{\prime}} y_{j}\right) \leq 0,
$$

where the first inequality follows from a combination of inequalities (18) $\Gamma$ and the second inequality follows from the assumption that $\left(q-\left|J^{\prime}\right|\right) \geq 0$.

Proof that (21) is facet defining:

Here we use the same technique as in the proof of Theorem 8. Let $\left|J^{\prime}\right|<\min \{n, q\}$. Without loss of generalityTassume that $J^{\prime} \ni 1$. Let $\alpha x+\beta y+\gamma z \leq \delta$ be a valid inequality for $P_{\mathrm{MC}^{\prime}}$ such that

$$
\begin{aligned}
& \mathcal{F} \equiv\left\{(x, y, z) \in P_{\mathrm{MC}^{\prime}}: \sum_{j \in J^{\prime}} y_{j}-\sum_{j \in J^{\prime}} \sum_{i \in I} \sum_{k \in K} x_{i j k}+\left(q-\left|J^{\prime}\right|\right)\left(1-\sum_{j \notin \cdot J^{\prime}} y_{j}\right)=0\right\} \\
& \subseteq\left\{(x, y, z) \in P_{\mathrm{MC}^{\prime}}: \sum_{i \in I} \sum_{j \in J} \sum_{k \in K} \alpha_{i j k} x_{i j k}+\sum_{j \in J} \beta_{j} y_{j}+\sum_{k \in K} \gamma_{k} z_{k}=\delta\right\} .
\end{aligned}
$$

For any $i^{\prime}, i^{\prime \prime} \in I, i^{\prime} \neq i^{\prime \prime}, j^{\prime} \notin J^{\prime}$ Tand $k^{\prime} \in K$ Tconsider the following two points in $\mathcal{F}$ :

$$
\begin{aligned}
x_{i j k} & = \begin{cases}1 & \text { if } i=i^{\prime} \text { and } j=j^{\prime} \Gamma \\
0 & \text { otherwise } \Gamma\end{cases} \\
y_{j} & = \begin{cases}1 & \text { if } j=j^{\prime} \Gamma \\
0 & \text { otherwise } \Gamma\end{cases} \\
z_{i} & = \begin{cases}1 & \text { if } i=i^{\prime} \Gamma \\
0 & \text { otherwise }\end{cases} \\
\text { and } \quad x_{i j k} & = \begin{cases}1 & \text { if } i=i^{\prime \prime} \Gamma j=j^{\prime} \text { and } k=k^{\prime} \Gamma \\
1 & \text { if } i=i^{\prime} \Gamma j=j^{\prime} \text { and } k \neq k^{\prime} \Gamma \\
0 & \text { otherwise } \Gamma\end{cases} \\
y_{j} & = \begin{cases}1 & \text { if } j=j^{\prime} \Gamma \\
0 & \text { otherwise } \Gamma\end{cases} \\
z_{i} & = \begin{cases}1 & \text { if } i=i^{\prime} \text { or } i^{\prime \prime} \Gamma \\
0 & \text { otherwise. }\end{cases}
\end{aligned}
$$

By comparing the two points we can conclude that

$$
\alpha_{i^{\prime} j^{\prime} k^{\prime}}=\alpha_{i^{\prime \prime} j^{\prime} k^{\prime}}+\gamma_{i^{\prime \prime}} \quad \text { for any } i^{\prime}, i^{\prime \prime} \in I, i^{\prime} \neq i^{\prime \prime} \text { Tany } j^{\prime} \notin J^{\prime} \text { Tand any } k^{\prime} \in K \text {. }
$$

Similarly for any $j^{\prime} \in J^{\prime} \Gamma$ and for any arbitrary $i^{\prime} \neq i^{\prime \prime}$ and any arbitrary $k^{\prime} \Gamma$ consider a solution where all clients are assigned to z-facility $i^{\prime}$ and y-facilities in $J^{\prime}$ such that all $\left|J^{\prime}\right|$ facilities are used $\Gamma$ and compare it to the solution where client $k^{\prime}$ is switched from z-facility $i^{\prime}$ to $i^{\prime \prime}$. We see that (23) also holds for $j \in J^{\prime}$. 
Next for $j^{\prime} \notin J^{\prime}$ Tcomparing point (22) and

$$
\begin{aligned}
x_{i j k} & = \begin{cases}1 & \text { if } i=i^{\prime \prime} \text { and } j=j^{\prime} \Gamma \\
0 & \text { otherwise } \Gamma\end{cases} \\
y_{j} & = \begin{cases}1 & \text { if } j=j^{\prime} \Gamma \\
0 & \text { otherwise } \Gamma\end{cases} \\
z_{i} & = \begin{cases}1 & \text { if } i=i^{\prime \prime} \Gamma \\
0 & \text { otherwise } \Gamma\end{cases}
\end{aligned}
$$

for any arbitrary $i^{\prime} \neq i^{\prime \prime}$ Tand using (23) we see that

$$
-(q-1) \gamma_{i^{\prime \prime}}=\gamma_{i^{\prime}} \quad \text { for any } i^{\prime} \neq i^{\prime \prime} .
$$

If $m \geq 3$ or $q \geq 3$ Tapplying (24) to all pairs of distinct $i^{\prime}$ and $i^{\prime \prime}$ shows that $\gamma_{i}=0$ for all $i$ Tand hence $\alpha_{i^{\prime} j k}=\alpha_{i^{\prime \prime} j k}$ for any $i \neq i^{\prime \prime}$ Tany $j$ Tand any $k$. When $m=q=2 \Gamma$ we have $\gamma_{2}=-\gamma_{1}$.

Next $\Gamma$ for any client pair $k^{\prime} \neq k^{\prime \prime} \Gamma$ and any $j^{\prime} \neq 1 \Gamma$ consider a point in $\mathcal{F}$ where client $k^{\prime}$ is assigned to $\mathrm{y}$-facility $1 \Gamma$ client $k^{\prime \prime}$ is assigned to $\mathrm{y}$-facility $j^{\prime}$ and all clients are assigned to z-facility 1 . Comparing this point to the point in $\mathcal{F}$ where the y-facilities for the clients $k^{\prime}$ and $k^{\prime \prime}$ are switched $\Gamma$ we get

$$
\begin{aligned}
\alpha_{11 k^{\prime}}+\alpha_{1 j^{\prime} k^{\prime \prime}} & =\alpha_{1 j^{\prime} k^{\prime}}+\alpha_{11 k^{\prime \prime}} \\
\text { or } \quad \alpha_{1 j^{\prime} k^{\prime}}-\alpha_{11 k^{\prime}} & =\alpha_{1 j^{\prime} k^{\prime \prime}}-\alpha_{11 k^{\prime \prime}} \quad \text { for any } k^{\prime} \neq k^{\prime \prime} .
\end{aligned}
$$

Therefore $\Gamma \alpha_{1 j k}=\alpha_{11 k}+\eta_{j}$ for some constant $\eta_{j}$ Tfor any $j \neq 1$ and any $k$.

NextTlet $i^{\prime}=1$. For any $k^{\prime} \Gamma$ compare (22) for $j^{\prime} \notin J^{\prime}$ and the following point:

$$
\begin{aligned}
x_{i j k} & = \begin{cases}1 & \text { if } i=i^{\prime} \Gamma j=j^{\prime \prime} \text { and } k=k^{\prime} \Gamma \\
1 & \text { if } i=i^{\prime} \Gamma j=j^{\prime} \text { and } k \neq k^{\prime} \Gamma \\
0 & \text { otherwise } \Gamma\end{cases} \\
y_{j} & = \begin{cases}1 & \text { if } j=j^{\prime} \text { or } j^{\prime \prime} \Gamma \\
0 & \text { otherwise } \Gamma\end{cases} \\
z_{i} & = \begin{cases}1 & \text { if } i=i^{\prime} \Gamma \\
0 & \text { otherwise. }\end{cases}
\end{aligned}
$$

For $j^{\prime \prime}=1$ we see that $\eta_{j}=\beta_{1}$ for all $j \notin J^{\prime}$. Compare (25) for $j^{\prime \prime}=1$ and $j^{\prime \prime} \in J^{\prime} \backslash\{1\} \Gamma$ we see that $\eta_{j}=\beta_{1}-\beta_{j}$ for all $j \in J^{\prime}$.

For any client $k^{\prime}$ Tconsider a point in $\mathcal{F}$ where all clients are assigned to z-facility $i$ and y-facilities in $J^{\prime}$ such that all $\left|J^{\prime}\right|$ facilities are used Tand such that client $k^{\prime}$ and at least one other client are assigned to $\mathrm{y}$-facility 1 . Compare this solution to the solution where client $k^{\prime}$ is re-assigned to some other y-facility $j^{\prime} \in J^{\prime}$. We can then conclude that $\alpha_{i 1 k}=\alpha_{i j k}$ for any $i$ Tany $j \in J^{\prime}$ Tand any $k$. Therefore $\eta_{j}=0$ Tand hence $\beta_{j}=\beta_{1}$ Tfor any $j \in J^{\prime}$. 
LastlyГlet $i^{\prime}=1$. For any $j_{1} \notin J^{\prime}$ and any $j_{2} \notin J^{\prime}$ with $j_{1} \neq j_{2} \Gamma$ comparing (22) for $j^{\prime}=j_{1}$ and $j^{\prime}=j_{2} \Gamma$ we see that $\beta_{j_{1}}=\beta_{j_{2}}$. Comparing (22) with $i^{\prime}=1, j^{\prime} \notin J^{\prime}$ and a point in $\mathcal{F}$ where all clients are assigned to z-facility 1 and y-facilities in $J^{\prime}$ Twe see that

$$
\beta_{j}=-(q-|J|) \beta_{1} \quad \text { for all } j \notin J^{\prime} .
$$

Substitution into (22) shows that $\delta=\sum_{k \in K} \alpha_{11 k}+\left|J^{\prime}\right| \beta_{1}+\gamma_{1}$. Therefore $\alpha x+\beta y+\gamma z \leq \delta$ can be written as

$$
\begin{gathered}
\sum_{k \in K}\left(\alpha_{11 k}+\beta_{1}\right) \sum_{j \in J} \sum_{k \in K} x_{i j k}-\gamma_{1}\left(z_{2}-\sum_{j \in J} \sum_{k \in K} x_{2 j k}\right) \\
+\beta_{1}\left(\sum_{j \in J^{\prime}} y_{j}-\sum_{j \in J^{\prime}} \sum_{i \in I} \sum_{k \in K} x_{i j k}+\left(q-\left|J^{\prime}\right|\right)\left(1-\sum_{j \notin J^{\prime}} y_{j}\right)\right) \\
\leq \sum_{k \in K}\left(\alpha_{11 k}+\beta_{1}\right)-\gamma_{1}\left(z_{1}-1\right) .
\end{gathered}
$$

When $m \geq 3$ or $q \geq 3 \Gamma \gamma_{1}=0$. Hence (21) represents a facet of $P_{\mathrm{MC}}$.

Corollary 13 Let $I^{\prime} \subseteq I$ with $1 \leq\left|I^{\prime}\right|<q$. The inequality

$$
\sum_{i \in I^{\prime}} z_{i}-\sum_{i \in I^{\prime}} \sum_{j \in J} \sum_{k \in K} x_{i j k}+\left(q-\left|I^{\prime}\right|\right)\left(1-\sum_{i \notin I^{\prime}} z_{i}\right) \leq 0
$$

is valid for $P_{\mathrm{MC}^{\prime}}$ and represents a facet if $\left|I^{\prime}\right|<\min \{m, q\}$.

Again $\Gamma$ if $m=q=2 \Gamma$ the inequality (26) for $\left|I^{\prime}\right|=1$ is an improper facet.

\section{Example 2}

As an illustration $\Gamma$ when $I=J=K=\{1,2,3\}$ Tthe following point defined by:

$$
\begin{aligned}
& x_{121}=\frac{1}{2}, \quad x_{211}=\frac{1}{6}, \quad x_{311}=\frac{1}{3}, \quad x_{212}=\frac{2}{3}, \quad x_{312}=\frac{1}{3}, \\
& x_{113}=\frac{1}{2}, \quad x_{223}=\frac{1}{6}, \quad x_{323}=\frac{1}{3}, \quad \text { all other } x_{i j k}=0, \\
& z_{1}=z_{2}=1, \quad z_{3}=\frac{1}{3}, \quad y_{1}=y_{2}=1, \quad y_{3}=0 .
\end{aligned}
$$

is in $\bar{X}_{\mathrm{MC}}$ but does not satisfy (26) with $I^{\prime}=\{1,2\}$.

We note that when $\left|I^{\prime}\right|=q-1$ and when $\left|J^{\prime}\right|=q-1$ the inequalities (21) and (26) are Chvátal-Gomory inequalities of rank 1 (see Nemhauser and WolseyГ1988). 


\subsubsection{Separation}

The separation problems based on the families of inequalities (21) and (26) are quadratic knapsack problems. HoweverTfor fixed size of the subsets $\left|I^{\prime}\right|$ and $\left|J^{\prime}\right|$ respectivelyГwe obtain linear 0-1 knapsack problems. Let $\left(x^{*}, y^{*}, z^{*}\right)$ denote a fractional solution to formulation $\mathrm{MC}^{\prime}$ and let $\pi_{j}=1$ if $j \in J^{\prime}$ and $\pi_{j}=0$ otherwise. Below we state the separation problem based on inequalities (21) for fixed $\left|J^{\prime}\right|=t$.

(KS):

$$
\max \sum_{j \in J}\left(y_{j}^{*}-\sum_{i \in I} \sum_{k \in K} x_{i j k}^{*}\right) \pi_{j}+(q-t)\left(1-\sum_{j \in J} y_{j}^{*}\left(1-\pi_{j}\right)\right)
$$

subject to

$$
\begin{aligned}
\sum_{j \in J} \pi_{j} & =t \\
\pi_{j} & \in\{0,1\}
\end{aligned}
$$

Since $y_{j}^{*}-\sum_{i \in I} \sum_{k \in K} x_{i j k}^{*} \leq 0$ for all $j \in J$ due to constraints (18) Tand since $q-t>0$ due to the assumption of Theorem 12 Tit is easy to see that inequality (21) can be violated only if $\sum_{j \notin J^{\prime}} y_{j}^{*}<1$. Let $J^{\prime \prime}$ denote a maximum cardinality subset of $J$ such that $\sum_{j \in J^{\prime \prime}} y_{j}^{*}<1$. It then follows that we only have to solve the knapsack problems KS for $n-\left|J^{\prime \prime}\right| \leq t \leq q-1$. Since the constraint coefficients of the $\pi_{j}$-variables are all equal to oneTproblem KS can be solved by a greedy algorithm.

\section{New Facets for the One-Level Uncapacitated Facility Lo- cation Problem}

All facet-defining inequalities for UFL previously known in the literature have $0-1$ coefficientsTexcept for facets obtained by simultaneous lifting (see Cornuéjols and ThizyГ 1982 and Cho et al.T1983b). In both classes of facet-defining inequalities introduced in Theorems 12 and 13 Tthe coefficients of $y_{j}$ for $j \notin J^{\prime}$ and $z_{i}$ for $i \notin I^{\prime}$ may be greater than one. If we consider the version of UFL where the constraints

$$
\sum_{k \in K} x_{j k} \geq y_{j} \quad \text { for all } j \in J,
$$

are added to the set of constraints defining $X_{\mathrm{UFL}}$ Twe obtain the following results. Let $X_{\mathrm{UFL}^{\prime}}$ be the set of solutions $(x, y)$ satisfying the constraints of $X_{\mathrm{UFL}}$ and constraint (27). Also let $P_{\mathrm{UFL}^{\prime}}=\operatorname{conv}\left(X_{\mathrm{UFL}^{\prime}}\right)$. 
Theorem 14 Let

$$
\sum_{j \in J} \sum_{k \in K} a_{j k} x_{j k}+\sum_{j \in J} b_{j} y_{j} \leq d,
$$

represent a facet of $P_{\mathrm{UFL}}$ that is not a non-negativity constraint for some $x_{i j}$. Then

$$
\sum_{j \in J} \sum_{k \in K} a_{j k} \sum_{i \in I} x_{i j k}+\sum_{j \in J} b_{j} y_{j} \leq d,
$$

represents a facet of $P_{\mathrm{MC}}$.

Theorem 15 Every facet of $P_{\mathrm{MC}^{\prime}}$ of the form

$$
\sum_{j \in J} \sum_{k \in K} a_{j k} \sum_{i \in I} x_{i j k}+\sum_{j \in J} b_{j} y_{j} \leq d,
$$

where the coefficient of $z_{i}$ is zero for all $i \in I$, and the coefficient of $x_{i j k}$ is independent of $i$ for all $i \in I, j \in J, k \in K$, induces a facet for $P_{\mathrm{UFL}^{\prime}}$.

The proofs of Theorems 14 and 15 are similar to the proofs of Theorems 5 and 6 and therefore omitted here.

By applying Theorem 15 to the facet-defining inequalities (21) and (26)Twe can obtain new facets for $P_{\mathrm{UFL}}$ Tthe modified formulation of the the one-level uncapacitated facility location problem.

\section{An Alternative Flow Formulation}

When solving integer or mixed-integer problems using LP-based solution techniques $\Gamma$ such as the cutting plane approach $\Gamma$ the choice of initial formulation plays an important role. It is crucial to obtain a good initial bound from the linear relaxation. On the other hand Tone wants to avoid too large formulations $\Gamma$ since it is practically inevitable that at least a few branch-and-bound nodes are needed to verify the optimal solution. If the formulation is very large the time gained from a very sharp LP-bound may be lost by the time consumed by solving a large linear program in every branch-and-bound node. A solution to this dilemma can be the use of a weakerTsmallerTinitial formulation and then augmenting the formulation by identifying and adding violated inequalitiesTthus in some way compensating for the lack of strength in certain parts of the initial formulation.

Computational experiments have shown that the linear programming bound produced by formulation MC in general is very sharp (see Rardin and ChoeГ 1979). However $\Gamma$ the formulation grows rapidly with the size of the instance. Therefore $\Gamma$ we introduce in the following subsection a weaker flow formulation $\Gamma$ as an alternative to the multi-commodity formulation MC. In Section 5.2 we discuss how the flow formulation can be strengthened by adding dicut collection inequalities (see Rardin and WolseyГ 1993). 


\subsection{The Single-Commodity Flow Formulation}

Let $w_{i j}$ be the quantity sent from z-facility $i$ to $\mathrm{y}$-facility $j$ and $v_{j k}$ the quantity sent from y-facility $j$ to client $k$. Note that $v_{j k}=\sum_{i \in I} x_{i j k}$ and $w_{i j}=\sum_{k \in K} x_{i j k}$. Without loss of generality we assume that each client's demand is one unit. The set of feasible solutions to the flow formulationTFTis given by the following sets of constraints:

$$
\text { (F) } \begin{aligned}
\sum_{j \in J} v_{j k} & =1 & & \text { for all } k \in I, \\
v_{j k} & \leq y_{j} & & \text { for all } j \in J, k \in K, \\
\sum_{k \in K} v_{j k}-\sum_{i \in I} w_{i j} & =0 & & \text { for all } j \in J, \\
\sum_{j \in J} w_{i j} & \leq q z_{i} & & \text { for all } i \in I, \\
v_{j k} & \geq 0 & & \text { for all } j \in J, k \in K, \\
w_{i j} & \geq 0 & & \text { for all } i \in I, j \in J, \\
0 \leq y_{j} & \leq 1 & & \text { for all } j \in J, \\
0 \leq z_{i} & \leq 1 & & \text { for all } i \in I, \\
z_{i}, y_{j}, \text { integer } & \text { valued } & & \text { for all } i \in I, j \in J .
\end{aligned}
$$

Let

$$
\begin{aligned}
& X_{\mathrm{F}}=\left\{(v, w, y, z) \in R^{n q+m n+m+n}:(v, w, y, z) \text { satisfies }(30)-(38)\right\}, \\
& \bar{X}_{\mathrm{F}}=\left\{(v, w, y, z) \in R^{n q+m n+m+n}:(v, w, y, z) \text { satisfies }(30)-(37)\right\},
\end{aligned}
$$

and let $P_{\mathrm{F}}=\operatorname{conv}\left(X_{\mathrm{F}}\right)$.

Proposition $16 \bar{X}_{\mathrm{MC}} \subset \bar{X}_{\mathrm{F}}$.

Proof. By using $v_{j k}=\sum_{i \in I} x_{i j k}$ and $w_{i j}=\sum_{k \in K} x_{i j k}$ Twe can easily verify that $\bar{X}_{\mathrm{F}}$ is

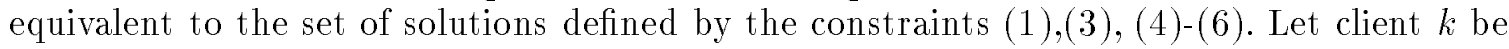
assigned to z-facility $i$ and $\mathrm{y}$-facility $j \Gamma$ and assume that all other clients are assigned to different facilities. $z_{i}=1 / q$ is feasible in $\bar{X}_{\mathrm{F}}$ Twhereas one of the constraints (2) in $\bar{X}_{\mathrm{MC}}$ would require $z_{i}=1$.

From the proof of Proposition 16 we observe that the flow formulation does not model the fixed-charge structure of the z-facility level as well as the MC formulation due to the weaker constraint set (33). In the next section we shall describe a class of inequalities that can be used to strengthen the flow formulation. 


\subsection{Dicut Collection Inequalities}

Rardin and Wolsey (1993) derived a class of valid inequalities $\Gamma$ called dicut collection inequalitiesTfor general uncapacitated fixed-charge problemsTfrom the projection of a multicommodity formulation onto the space of the flow variables. In fact Tthey showed that the class of dicut collection inequalities precisely describes this projection. They also showed that these inequalities subsume many of the previously-known inequalities developed for more specific network problems $\Gamma$ such as uncapacitated lot sizing.

Here we describe a subclassTcalled simple dicut collection inequalities. Without loss of generality Rardin and Wolsey (1993) consider a directed graph with only one source node $s$ and a set of sink nodes $T$ Twhere the set of arcs are partitioned into a set of continuous $\operatorname{arcs} C$ and a set of fixed-charge $\operatorname{arcs} F$. A $t$-dicut for $t \in T$ is a set $R$ of arcs whose removal will block the flow from $s$ to $t$. We assume that each $t$-dicut is minimal.

Let $\Gamma=\left\{\Gamma^{t}\right\}_{t \in T}$ be a collection of sets of dicuts $\Gamma$ with no more that one dicut in each set for each sink. Let $\gamma^{t}=\left|\Gamma^{t}\right|$; hence $\Gamma \gamma^{t} \leq 1$ for all $t \in T$. Moreover $\Gamma$ let $\gamma_{e}=1$ if arc $e$ is in some dicut in $\Gamma$ and $\gamma_{e}=0$ otherwise. Furthermore $\Gamma$ let

$$
\begin{aligned}
& x_{e}=\text { the flow on } \operatorname{arc} e \in C, \\
& y_{e}= \begin{cases}1 & \text { if } \operatorname{arc} e \in F \text { is open } \Gamma \\
0 & \text { otherwise } \Gamma\end{cases}
\end{aligned}
$$

and let $d_{t}$ denote the demand of sink $t$. The corresponding simple dicut collection inequality is:

$$
\sum_{e \in C} \gamma_{e} x_{e}+\sum_{e \in F} \sum_{t \in T} d_{t} \gamma_{e} y_{e} \geq \sum_{t \in T} \gamma^{t} d_{t} .
$$

To represent the two-level facility location problem as a fixed-charge network of the form considered by Rardin and Wolsey (1993) Twe introduce a source node $s$ and arcs from $s$ to every $z$-facility $i$. We also split vertices representing facilities $i$ and $j$ into two vertices $i, i^{\prime}$ and $j^{\prime}, j$ respectively. The set of sink vertices is the set $K$ of clients. The $\operatorname{arcs}\left(j^{\prime}, j\right)$ for all $j \in J$ and $\left(i, i^{\prime}\right)$ for all $i \in I$ are the fixed-charge arcs of the network. The arcs $(s, i)$ for all $i \in I \Gamma\left(i^{\prime}, j^{\prime}\right)$ for all $i \in I, j \in J$ Tand $(j, k)$ for all $j \in J, k \in K$ are the continuous arcs. The values of $v_{j k}$ and $w_{i j}$ in formulation $\mathrm{F}$ represent flows on $\operatorname{arcs}(j, k)$ and $\left(i^{\prime}, j^{\prime}\right)$ respectively. The structure of the digraph relevant to TUFL is shown in Figure 2.

One subclass of the simple dicut collection inequalities that seems particularly useful for formulation F of TUFL is the so-called fixed-charge path inequalities (see Van Roy and Wolsey 1987 ). These inequalities involve variables for both $\mathrm{z}$ - and $\mathrm{y}$-facilities as well as clients.

Consider the path $(\tilde{i}, \tilde{j}, \tilde{k})$ from $z$-facility $\tilde{i}$ Through y-facility $\tilde{j}$ to client $\tilde{k}$. The demand of the nodes in the path is equal to zero except for client $\tilde{k}$. HenceTif we choose a subset 


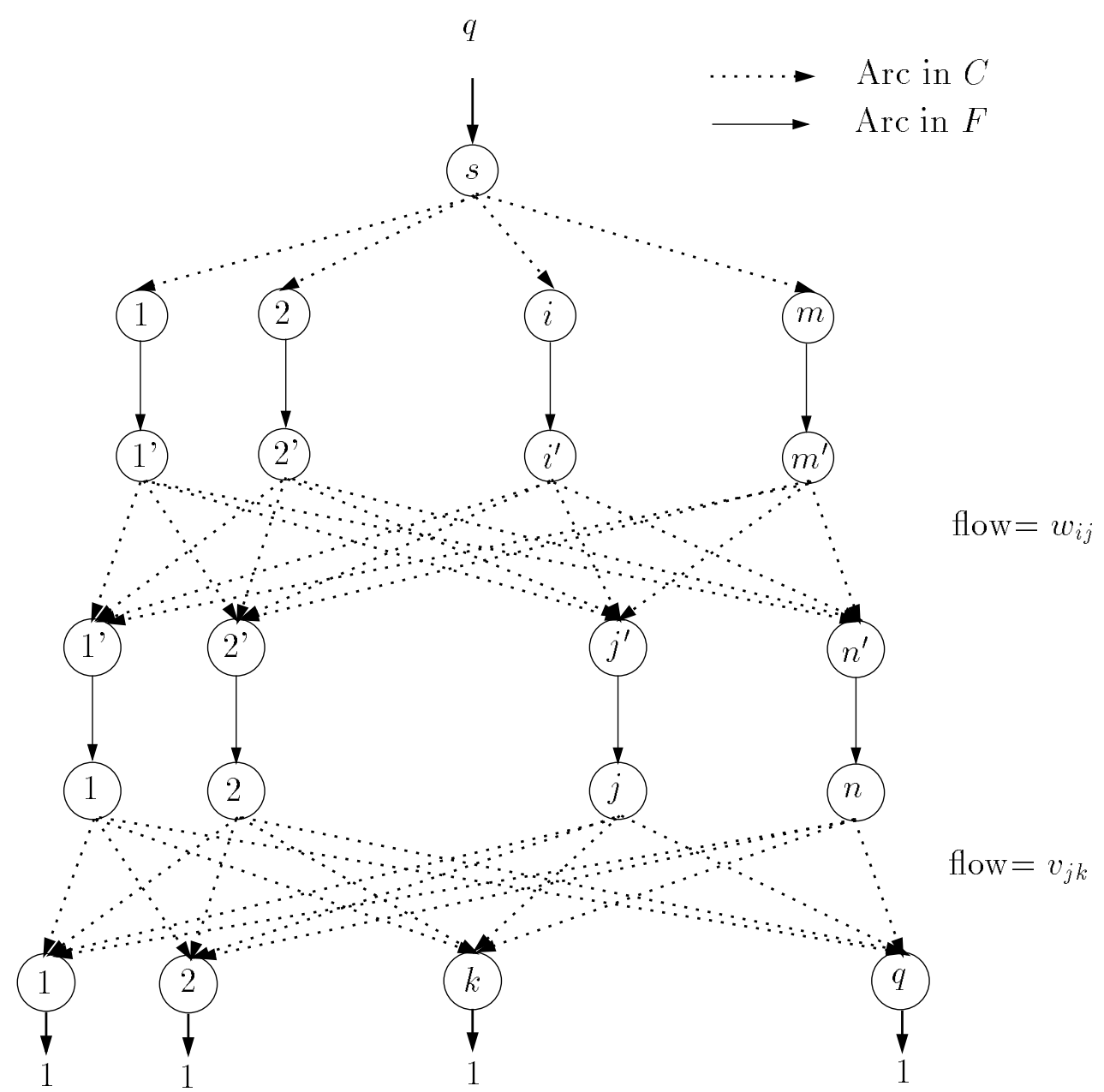

Figure 2: TUFL as a fixed-charge network flow problem.

of arcs supplying any of the nodes on the path Tthen the total flow on these arcs has to be less than or equal to the demand of client $\tilde{k}$ plus all possible outflow from that node and all nodes further down on the path. For the inflow arcs we make use of the fixed-charge structure. The path structure is shown in Figure 3.

Let $I^{+} \subseteq I \backslash\{\tilde{i}\}$ Tand $J^{+} \subseteq J \backslash\{\tilde{j}\}$. Also $\Gamma$ let $w_{i}$ denote the flow from the source $s$ to $z$-facility $i$. The fixed-charge path inequality for path $(\tilde{i}, \tilde{j}, \tilde{k})$ is:

$$
w_{\tilde{i}}+\sum_{i \in I^{+}} w_{i \tilde{j}}+\sum_{j \in J^{+}} v_{j \tilde{k}} \leq d_{\tilde{k}} z_{\tilde{i}}+\sum_{i \in I^{+}} d_{\tilde{k}} z_{i}+\sum_{j \in J^{+}} d_{\tilde{k}} y_{j}+\sum_{j \in(J \backslash\{\tilde{j}\})} w_{\tilde{i} j}+\sum_{k \in K \backslash\{\tilde{k}\}} v_{\tilde{j} k}
$$

Note that $d_{k}=1$ for all $k \in K$ in our case. The following example shows how an inequality of type (40) can be used to cut off a fractional solution of $\bar{X}_{\mathrm{F}}$. 


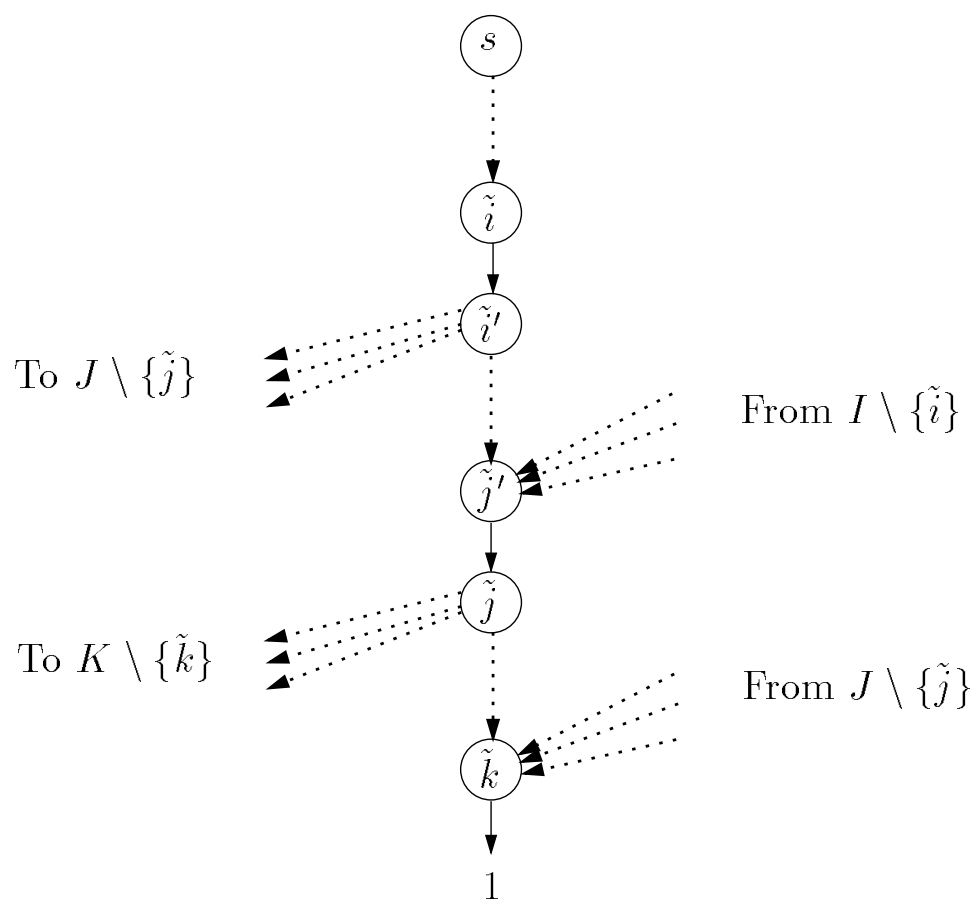

Figure 3: Structure of a path inequality.

\section{Example 3}

Let $I=J=K=\{1,2,3\}$ and consider the path $(\tilde{i}=1, \tilde{j}=1, \tilde{k}=1)$ with $I^{+}=\{3\}$ and $J^{+}=\{3\}$. Then the point in $\bar{X}_{\mathrm{F}}$ defined by:

$$
\begin{aligned}
& z_{1}=\frac{1}{3}, \quad z_{2}=\frac{2}{3}, \quad z_{3}=0, \quad y_{1}=y_{2}=1, \quad y_{3}=0, \\
& w_{11}=1, \quad w_{22}=2, \quad \text { all other } w_{i j}=0, \\
& v_{11}=1, \quad v_{22}=1, \quad v_{23}=1, \quad \text { all other } v_{j k}=0,
\end{aligned}
$$

does not satisfy the path inequality:

$$
\underbrace{w_{11}+w_{12}+w_{13}}_{w_{1}}+w_{31}+v_{31} \leq z_{1}+z_{3}+y_{3}+w_{12}+w_{13}+v_{12}+v_{13} .
$$

To see that the inequality (40) is a simple dicut inequality derived from flow balance along the path $(\tilde{i}, \tilde{j}, \tilde{k})$ :

$$
w_{i}+\sum_{i \in I^{+}} w_{i \tilde{j}}+\sum_{j \in J^{+}} v_{j \tilde{k}}=d_{\tilde{k}}+\sum_{j \in J \backslash\{\tilde{j}\}} w_{\tilde{i} j}+\sum_{k \in K \backslash\{\tilde{k}\}} v_{\tilde{j} k}-\sum_{i \in(I \backslash\{\tilde{i}\}) \backslash I^{+}} w_{i \tilde{j}}-\sum_{j \in(J \backslash\{\tilde{j}\}) \backslash J^{+}} v_{j \tilde{k}} .
$$


Substituting in $(40)$ gives

$$
\sum_{i \in(I \backslash\{\tilde{i}\}) \backslash I^{+}} w_{i \tilde{j}}+\sum_{j \in(J \backslash\{\tilde{j}\}) \backslash J^{+}} v_{j \tilde{k}}+d_{\tilde{k}} z_{\tilde{i}}+\sum_{i \in I^{+}} d_{\tilde{k}} z_{i}+\sum_{j \in J^{+}} d_{\tilde{k}} y_{j} \geq d_{\tilde{k}},
$$

which is exactly the simple dicut collection inequality where $\Gamma^{k}=\emptyset$ for all $k \in K \backslash\{\tilde{k}\}$ and

$$
\begin{aligned}
\Gamma^{\tilde{k}}= & \left\{\left(i, i^{\prime}\right): i \in\{\tilde{i}\} \cup I^{+}\right\} \cup\left\{\left(j^{\prime}, j\right): j \in J^{+}\right\} \\
& \cup\left\{\left(i^{\prime}, \tilde{j}\right): i \notin\{\tilde{i}\} \cup I^{+}\right\} \cup\left\{(\tilde{i}, j): j \notin\{\tilde{j}\} \cup J^{+}\right\} .
\end{aligned}
$$

Since the flow on the arc from a $y$-facility to a client cannot exceed the client's demand $\Gamma$ we can extend the path inequality to the following valid inequality:

$$
\begin{aligned}
w_{\tilde{i}}+\sum_{i \in I^{+}} w_{i j}+\sum_{j \in J^{+}} v_{j \tilde{k}} \leq & \left(\sum_{k \in K^{-}} d_{k}+d_{\tilde{k}}\right) z_{\tilde{i}}+\sum_{i \in I^{+}}\left(\sum_{k \in K^{-}} d_{k}+d_{\tilde{k}}\right) z_{\tilde{i}} \\
& +\sum_{j \in J^{+}} d_{\tilde{k}} y_{j}+\sum_{j \in(J \backslash\{\tilde{j}\})} w_{i j}+\sum_{k \in(K \backslash\{\tilde{k}\}) \backslash K^{-}} v_{\tilde{j} k}
\end{aligned}
$$

where $K^{-} \subseteq K \backslash\{\tilde{k}\}$.

The separation problem based on the dicut collection inequalities (39) can be solved by checking if a fractional solution $\left(w^{*}, v^{*}, y^{*}, z^{*}\right)$ of the single-commodity flow formulation is feasible for the multi-commodity extended formulation. That is $\Gamma$ if there is a solution $\left(x^{*}, y^{*}, z^{*}\right)$ for MC such that $\sum_{i \in I} x_{i j k}^{*}=v_{j k}^{*}$ and $\sum_{k \in K} x_{i j k}^{*}=w_{i j}^{*}$ for all $i \in I \Gamma j \in J$ and $k \in K$. If there is $\Gamma$ then all dicut collection inequalities are satisfied; if notTone has to examine the dual of the multi-commodity extended formulation $\Gamma$ which is a large LP for any reasonable-sized problem $\Gamma$ to identify a violated inequality. No combinatorial algorithm for solving the separation problem is known. A heuristic for finding violated fixed-charge path inequalities (40) and extended fixed-charge path inequalities (42) can be found in Van Roy and Wolsey (1987).

\section{$6 \quad$ Extensions}

Rardin and Choe (1979) have shown that the multi-commodity extended formulation models the fixed-charge behavior more carefully than single-commodity flow models. They presented empirical evidence showing that the LP-relaxation of the multi-commodity flow formulation is usually tight and is provably exact in some special networks. Since the model MC is equivalent to a multi-commodity flow formulation of the two-level uncapacitated location problem $\Gamma$ we should expect its $L P$-relaxation to be relatively tight. Indeed $\Gamma$ the largest gap that we have been able to construct for Euclidean problems as an example is 9 percent. 
We plan to develop and test a cutting plane approach to solving the two-level uncapacitated facility location problem $\mathrm{u}$ sing the models $\mathrm{MC}$ and $\mathrm{MC}^{\prime}$. One question to address is the effectiveness of the facets introduced in Section 3. Another issue to be explored is the tradeoff between the model size and solution quality between the stronger and weaker flow formulations.

\section{Acknowledgements}

The first author is most grateful for the financial support given by David ShmoysTCornell University through NSF grant CCR-9307391. The second author is an NFSR Research Associate. The work of the third author is partially supported by a grant from the University of Arizona Foundation Tand that of the fourth author by a research grant from the National Sciences and Engineering Research Council (NSERC) of Canada.

\section{References}

K. AardatT1992. On the Solution of One and Two-Level Capacitated Facility Location

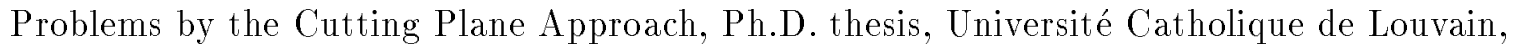
Louvain-la-NeuveГ Belgium.

A. Balakrishnan and S.C. GravesT1989. A Composite Algorithm for a Concave-Cost Network Flow Problem. Networks 19, 175-202.

A.I. Barros and M. LabiéT1992. A General Model for the Uncapacitated Facility and

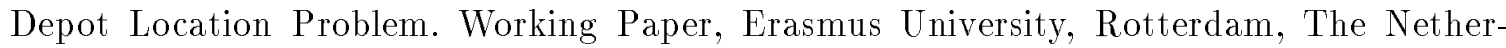
lands.

D.C. Cho, E.L. Johnson, M. Padberg and M.R. RaoT1983a. On the Uncapacitated Plant Location Problem I: Valid Inequalities and Facets. Mathematics of Operations Research 8, 579-589.

D.C. Cho, M. Padberg and M.R. RaoT1983b. On the Uncapacitated Plant Location Problem II: Facets and Lifting Theorems. Mathematics of Operations Research 8, 590-612.

G. Cornuédols, M. Fisher and G.L. Nemhauser T1977. On the Uncapacitated Location Problem. Annals of Discrete Mathematics 1, 163-177.

G. Cornuejols, G.L. Nemhauser and L.A. WolseyT1990. The Uncapacitated Facility Location Problem. Chapter 3 in P.B. Mirchandani and R.L. Francis (eds.) TDiscrete Location Theory WWileyГpp. 119-172. 
G. Cornuéuols and J.-M. ThizyT1982. Some Facets of the Simple Plant Location Polytope. Mathematical Programming 23, 50-74.

M.A. Efroymson and T.L. RayT1966. A Branch-Bound Algorithm for Plant Location. Operations Research 14, 361-368.

D. ErlenkotterT1978. A Dual-Based Procedure for Uncapacitated Facility Location. Operations Research 26, 992-1009.

M. Guignard 1980 . Fractional Vertices $\Gamma$ Cuts and Facets of the Simple Plant Location Problem. Mathematical Programming 12, 150-162.

L. Kaufman, M. Vanden Eede and P. HansenT1977. A Plant and Warehouse Location Problem. Operational Research Quarterly 28, 547-557.

G. Nemhauser and L. WolseyT1988. Integer and Combinatorial Optimization. Wiley \& Sons Inc.

R.L. Rardin and U. СhoeГ1979. Tighter Relaxations of Fixed Charge Network Flow Problems Report J-79-18ГIndustrial and Systems EngineeringГ Georgia Institute of Tech-

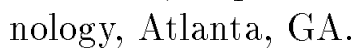

R.L. Rardin and L.A. WolseyT1993. Valid Inequalities and Projecting the Multicommodity Extended Formulation for Uncapacitated Fixed Charge Network Flow Problems. European Journal of Operational Research 71, 95-109.

D. Tcha And B. LeeT1984. A Branch-and-Bound Algorithm for the Multi-Level Uncapacitated Facility Location Problem. European Journal of Operational Research 18, 35-43.

T.J. Van Roy and L.A. WolseyT1987. Solving Mixed Integer Programming Problems Using Automatic Formulation. Operations Research 35, 45-57. 


\section{Appendix}

Theorem $3(x, y, z) \in \bar{X}_{\mathrm{MC}}$ is an extreme point of $\bar{X}_{\mathrm{MC}}$ if and only if

(a) For all $i \in I_{1}, z_{i}=\max _{k} \sum_{j \in J} x_{i j k}$.

(b) For all $j \in J_{1}, y_{j}=\max _{k} \sum_{i \in I} x_{i j k}$.

(c) for any $k$ in $K_{1}$, the graph $\hat{G}_{k}$ does not contain any even undirected circuit,

(d) there is no non-zero vector $\left(\alpha^{\prime}, \beta^{\prime}\right) \in \mathbf{R}^{I_{1}} \times \mathbf{R}^{J_{1}}$ such that:

for each $k \in K_{1}$, there is a pseudo-feasible flow $f^{k}$ for $G_{k}$ with $f_{(s, i)}^{k}=$ $\alpha_{i}^{\prime}$ for all $i \in \tilde{I}_{k} \cap I_{1}$ and $f_{(j, t)}^{k}=\beta_{j}^{\prime}$ for all $j \in \tilde{J}_{k} \cap J_{1}$.

The vector $\left(\alpha^{\prime}, \beta^{\prime}\right)$ specifies a set of flow-values for the arcs incident to the source node $s$ and sink node $t$ that is held fixed for all the graphs $G_{k}, k \in K_{1}$. Condition (8) says that for each $k \in K_{1}$ Twe can assign flow-values to the remaining arcs in $G_{k}$ to give a pseudo-feasible flow.

Proof. It is easy to check that $(x, y, z)$ is not extreme if Conditions (a) and (b) are violated. Suppose condition (c) is violated and the graph $\tilde{G}_{k}$ contain an even undirected circuit. In the first case $\Gamma$ suppose this circuit does not contain node $d$. Then $\Gamma$ the circuit contains the arcs:

$$
\left\{\left(i_{1}, j_{1}\right),\left(i_{2}, j_{1}\right),\left(i_{2}, j_{2}\right)\left(i_{3}, j_{2}\right) \ldots,\left(i_{s}, j_{s}\right),\left(i_{1}, j_{s}\right)\right\}
$$

for some $s \geq 2$. Note that the values of the $x_{i j k}$ 's corresponding to these arcs are strictly between 0 and 1 . Construct a point $\left(x^{\prime}, y, z\right)$ by alternately increasing and decreasing the corresponding $x_{i j k}$ 's for the sequence of the arcs in this circuit $\Gamma$ that is

$$
\begin{aligned}
& x_{i_{h} j_{h} k}^{\prime}=x_{i_{h} j_{h} k}+\epsilon^{\prime} \quad \text { for } h=1, \ldots, s, \\
& x_{i_{h+1} j_{h} k}^{\prime}=x_{i_{h+1} j_{h} k}-\epsilon^{\prime} \quad \text { for } h=1, \ldots, s \text {, with } i_{s+1}=i_{1}, \\
& \text { and all other elements of }\left(x^{\prime}, y, z\right) \text { the same as }(x, y, z) \text {, }
\end{aligned}
$$

for some $\epsilon^{\prime}>0$. A similar point $\left(x^{\prime \prime}, y, z\right)$ can be constructed with $\epsilon^{\prime \prime}=-\epsilon^{\prime}$. Note that

$$
\begin{array}{rlrl}
\sum_{j \in J} x_{i j k}^{\prime} & =\sum_{j \in J} x_{i j k}^{\prime \prime}=\sum_{j \in J} x_{i j k} & & \forall i \in I, k \in K, \\
\text { and } \quad & \sum_{i \in I} x_{i j k}^{\prime}=\sum_{i \in I} x_{i j k}^{\prime \prime}=\sum_{i \in I} x_{i j k} \quad & \forall j \in J, k \in K .
\end{array}
$$


For a sufficiently small $\epsilon^{\prime}$ Tboth points $\left(x^{\prime}, y, z\right)$ and $\left(x^{\prime \prime}, y, z\right)$ are in $\bar{X}_{\mathrm{MC}} \Gamma$ showing that $(x, y, z)$ is not an extreme point of $\bar{X}_{\mathrm{MC}}$.

In the second case $\Gamma$ suppose the circuit contains node $d$. Since the circuit is even $\Gamma$ the number of arcs not incident to $d$ is also even. Again we can construct a point $\left(x^{\prime}, y, z\right)$ by alternately increasing and decreasing by $\epsilon^{\prime}>0$ the $x_{i j k}$ 's corresponding to the sequence of arcs of the circuit Tskipping the arcs incident to $d$. An illustration is shown in Figure 4.

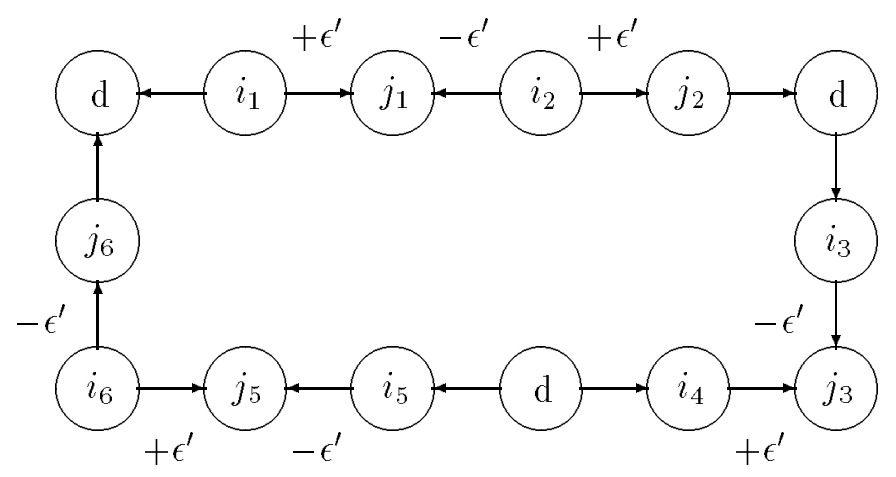

Figure 4: Using an even circuit to construct the point $\left(x^{\prime}, y, z\right)$.

For node $i$ incident from $d$ in the circuit $\Gamma \sum_{j \in J} x_{i j k}^{\prime}$ may differ from $\sum_{j \in J} x_{i j k}$ Tand for node $j$ incident to $d$ in the circuit $\Gamma \sum_{i \in I} x_{i j k}^{\prime}$ may differ from $\sum_{i \in I} x_{i j k}$. However $\Gamma$ since the arcs $(d, i)$ and $(j, d)$ in $G_{k}$ indicate that the corresponding constraints $(2)$ and $(3)$ are slack $\Gamma$ $\left(x^{\prime}, y, z\right)$ will still be in $\bar{X}_{\mathrm{MC}}$ if $\epsilon^{\prime}$ is sufficiently small. As in the first case a similar point $\left(x^{\prime \prime}, y, z\right)$ with $\epsilon^{\prime \prime}=-\epsilon^{\prime}$. Since $(x, y, z)$ is a convex combination of $\left(x^{\prime}, y, z\right)$ and $\left(x^{\prime \prime}, y, z\right)$ Tand by choosing $\epsilon^{\prime}>0$ Tbut small enough $\Gamma$ both $\left(x^{\prime}, y, z\right)$ and $\left(x^{\prime \prime}, y, z\right)$ will be in $\bar{X}_{\mathrm{MC}}$ Tthen $(x, y, z)$ is not an extreme point.

Finally $\Gamma$ we will show that if Conditions $(\mathbf{a}) \Gamma(\mathbf{b})$ and $(\mathbf{c})$ are satisfied $\Gamma(x, y, z)$ is an extreme point of $\bar{X}_{\mathrm{MC}}$ if and only if Condition (d) holds.

$$
\text { Let } \begin{aligned}
I_{2} & =\left\{i: z_{i}=0\right\}, \quad I_{3}=\left\{i: z_{i}=1\right\}, \\
J_{2} & =\left\{j: y_{j}=0\right\}, \quad J_{3}=\left\{j: y_{j}=1\right\}, \\
K_{2} & =\left\{k: x_{i j k} \text { integer-valued for all } i \text { and all } j\right\} .
\end{aligned}
$$

Note that $I_{1} \Gamma I_{2}$ and $I_{3}$ partition $I \Gamma J_{1}, J_{2}$ and $J_{3}$ partition $J \Gamma$ and $K_{1} \Gamma K_{2}$ partition $K$.

Suppose condition $(\mathbf{d})$ is violated. Consider $\left(x^{\prime}, y^{\prime}, z^{\prime}\right)$ and $\left(x^{\prime \prime}, y^{\prime \prime}, z^{\prime \prime}\right)$ where

$$
\begin{array}{rlrl}
z_{i}^{\prime} & =z_{i}+\alpha_{i}^{\prime}, & y_{j}^{\prime}=y_{j}+\beta_{j}^{\prime}, & x_{i j k}^{\prime}=x_{i j k}+\gamma_{i j k}^{\prime}, \\
\text { and } \quad z_{i}^{\prime \prime}=z_{i}-\alpha_{i}^{\prime}, & y_{j}^{\prime \prime}=y_{j}-\beta_{j}^{\prime}, & x_{i j k}^{\prime \prime}=x_{i j k}-\gamma_{i j k}^{\prime} .
\end{array}
$$


By considering the upper and lower bounds on $z_{i} \Gamma y_{j}$ and $x_{i j k} \Gamma$ we see that $\alpha_{i}^{\prime}=0$ for $i \in I_{2} \cup I_{3}$ and $\beta_{j}^{\prime}=0$ for $j \in J_{2} \cup J_{3}$ in order for $\left(x^{\prime}, y^{\prime}, z^{\prime}\right)$ and $\left(x^{\prime \prime}, y^{\prime \prime}, z^{\prime \prime}\right)$ to be in $\bar{X}_{\mathrm{MC}}$. FurthermoreTwe must have

$$
\begin{array}{ll}
\gamma_{i j k}^{\prime}=0 \quad & \text { for } i \in I_{1} \cup I_{2} \cup I_{3}, \quad j \in J_{2}, \quad k \in K_{1}, \\
& \text { for } i \in I_{2}, \quad j \in J_{1} \cup J_{2} \cup J_{3}, \quad k \in K_{1}, \quad \text { and } \\
& \text { for } i \in I_{1} \cup I_{2} \cup I_{3}, \quad j \in J_{1} \cup J_{2} \cup J_{3}, \quad k \in K_{2} .
\end{array}
$$

This leaves $\alpha_{i}^{\prime}$ for $i \in I_{1} \Gamma \beta_{j}^{\prime}$ for $j \in J_{1}$ and $\gamma_{i j k}^{\prime}$ for $i \in I_{1} \cup I_{3}, j \in J_{1} \cup J_{3}, k \in K_{1}$ undetermined.

Let $\left(\alpha^{\prime}, \beta^{\prime}\right)$ satisfy (8)Tand let $f^{k}$ for $k \in K_{1}$ be a corresponding set of pseudo-feasible flows. Then the values for $\gamma_{i j k}^{\prime}$ can be determined as follows. For $k \in K_{1}$ Twe define

$$
\gamma_{i j k}^{\prime}= \begin{cases}0 & \text { if } x_{i j k}=0 \text { or } 1 \Gamma \\ f_{(i, j)}^{k} & \text { if } 0<x_{i j k}<1\end{cases}
$$

Note that flow balance at the nodes $\tilde{I}_{k} \cap I_{1}$ and $\tilde{J}_{k} \cap J_{1}$ ensures that $\sum_{j \in J} \gamma_{i j k}^{\prime}=\alpha_{i}^{\prime}$ and $\sum_{i \in I} \gamma_{i j k}^{\prime}=\beta_{j}^{\prime}$ if the corresponding constraints (2) and (3) are tight. Flow balance at the nodes $s$ and $t$ ensures that $\sum_{\tilde{I}_{k} \cap I_{1}} \alpha_{i}^{\prime}=0$ and $\sum_{\tilde{J}_{k} \cap J_{1}} \beta_{j}^{\prime}=0 \Gamma$ which are also necessary for feasibility. FurtherTwe note that the graphs $G_{k}$ for $k \in K_{1}$ are of two types:

1. $\hat{G}_{k}$ contains no undirected cycles - whence any pseudo-feasible flow satisfies $\sum_{i \in I} \sum_{j \in J} \gamma_{i j k}^{\prime}=0$.

2. $\hat{G}_{k}$ contains an undirected odd cycle - in this case $\Gamma$ by adjusting the flow on the sub-paths in the cycles that start and end with $d$ and contain an odd number of $\operatorname{arcs} \Gamma$ a pseudo-feasible flow can be constructed with $\sum_{i \in I} \sum_{j \in J} \gamma_{i j k}^{\prime}=0$.

We also note that we can always assume that $\left(\alpha^{\prime}, \beta^{\prime}\right)$ is scaled such that

$$
\begin{aligned}
\left|\alpha_{i}^{\prime}\right| & \leq \min \left\{z_{i}, 1-z_{i}\right\} \quad \text { for all } i \in I_{1}, \\
\left|\beta_{j}^{\prime}\right| & \leq \min \left\{y_{j}, 1-y_{j}\right\} \quad \text { for all } j \in J_{1}, \\
\text { and } \quad\left|f_{\left(i^{\prime}, j^{\prime}\right)}^{k}\right| & \leq \min \left\{x_{i^{\prime} j^{\prime} k}, 1-x_{i^{\prime} j^{\prime} k}\right\} \quad \text { for }\left(i^{\prime}, j^{\prime}\right) \text { in the odd cycles in any } G^{k} .
\end{aligned}
$$

Thus $\mathrm{if}$ condition (d) does not hold $\mathrm{d}$ then the values of $\alpha^{\prime} \Gamma \beta^{\prime}$ and $\gamma^{\prime}$ can be set as above to show that $(x, y, z)$ is a convex combination of two distinct points in $\bar{X}_{\mathrm{MC}}$.

Now suppose condition (d) holdsTand suppose that the two points as defined by (43) are in $\bar{X}_{\mathrm{MC}}$. By considering the upper and lower bounds on the variables $\Gamma$ we again see that $\alpha_{i}^{\prime}=0$ for $i \in I_{2} \cup I_{3}$ and $\beta_{j}^{\prime}=0$ for $j \in J_{2} \cup J_{3}$ Tand the conditions (44) hold.

Now $\mathrm{Tin}$ order for the two points $\left(x^{\prime}, y^{\prime}, z^{\prime}\right)$ and $\left(x^{\prime \prime}, y^{\prime \prime}, z^{\prime \prime}\right)$ to be feasible for MCT we must have $\sum_{j \in J} \gamma_{i j k}^{\prime}=\alpha_{i}^{\prime}$ if the corresponding constraint (2) is tight; similarly $\Gamma \sum_{i \in I} \gamma_{i j k}^{\prime}=$ 
$\beta_{j}^{\prime}$ for $j \in \tilde{J}_{k} \cap J_{1}$ if the corresponding constraint (3) is tight. Further $\Gamma$ we must have $\sum_{i \in I} \sum_{j \in J} \gamma_{i j k}^{\prime}=0$ for every $k$ Timplying also $\sum_{i \in I} \alpha_{i}^{\prime}=0$ and $\sum_{j \in J} \beta_{j}^{\prime}=0$. We argue that $\alpha^{\prime} \Gamma \beta^{\prime}$ and $\gamma^{\prime}$ must be zero $\Gamma$ otherwise a non-zero vector $\left(\alpha^{\prime}, \beta^{\prime}\right)$ and corresponding pseudo-feasible flows can be constructed that violates (d). 